Network Working Group

Request for Comments: 2758

K. White

Category: Experimental

IBM Corp.

February 2000

\title{
Definitions of Managed Objects for Service Level \\ Agreements Performance Monitoring
}

Status of this Memo

This memo defines an Experimental Protocol for the Internet

community. It does not specify an Internet standard of any kind.

Discussion and suggestions for improvement are requested.

Distribution of this memo is unlimited.

Copyright Notice

Copyright (C) The Internet Society (2000). All Rights Reserved.

\section{Abstract}

This memo defines a Management Information Base (MIB) for performance monitoring of Service Level Agreements (SLAs) defined via policy definitions. The MIB defined herein focuses on defining a set of objects for monitoring SLAs and not on replication of the content of the policy definitions being monitored. The goal of the MIB defined within this document is to defined statistics related to a policy rule definition for reporting on the effect that a policy rule has on a system and to defined a method of monitoring this data.

Table of Contents

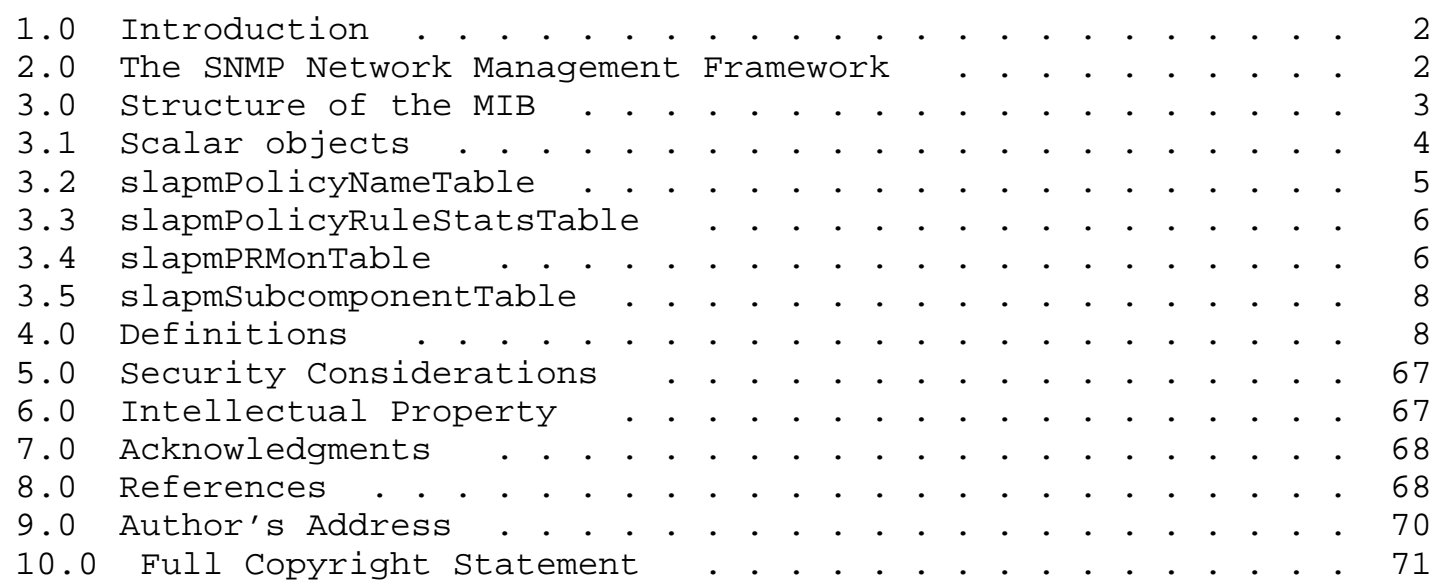




\subsection{Introduction}

The key words "MUST", "MUST NOT", "REQUIRED", "SHALL", "SHALL NOT", "SHOULD", "SHOULD NOT", "RECOMMENDED", "MAY", and "OPTIONAL" in this document are to be interpreted as described in RFC 2119, reference [13].

This document's purpose is to define a MIB module for performance management of Service Level Agreements (SLAs). It is assumed that an SLA is defined via policy schema definitions. The policy definitions being modeled with respect to performance management is primarily related to network Quality of Service (QOS). There are a number of methods that exist for defining and administering policy. Definition of these methods is considered out side of the scope of this document.

The MIB module defined within this memo has been modeled using the various versions of the schema definitions being developed within the Policy Framework Working Group in the IETF. The content of the MIB defined within this memo has evolved along with the Policy Framework Working Group schema definitions.

\subsection{The SNMP Network Management Framework}

The SNMP Management Framework presently consists of five major components:

- An overall architecture, described in RFC 2571 [7].

- Mechanisms for describing and naming objects and events for the purpose of management. The first version of this structure of Management Information (SMI) is called SMIv1 and described in STD 16, RFC 1155 [14], STD 16, RFC 1212 [15] and RFC 1215 [16]. The second version, called SMIv2, is described in STD 58, RFC 2578 [3], STD 58, RFC 2579 [4] and STD 58, RFC 2580 [5].

- Message protocols for transferring management information. The first version of the SNMP message protocol is called SNMPv1 and described in STD 15, RFC 1157 [1]. A second version of the SNMP message protocol, which is not an Internet standards track protocol, is called SNMPV2c and described in RFC 1901 [17] and RFC 1906 [18]. The third version of the message protocol is called SNMPv3 and described in RFC 1906 [18], RFC 2572 [8] and RFC 2574 [10].

- Protocol operations for accessing management information. The first set of protocol operations and associated PDU formats is described in STD 15, RFC 1157 [1]. A second set of protocol 
operations and associated PDU formats is described in RFC 1905 [6].

- A set of fundamental applications described in RFC 2573 [9] and the view-based access control mechanism described in RFC 2575 [11].

Managed objects are accessed via a virtual information store, termed the Management Information Base or MIB. Objects in the MIB are defined using the mechanisms defined in the SMI.

This memo specifies a MIB module that is compliant to the SMIV2. A MIB conforming to the SMIv1 can be produced through the appropriate translations. The resulting translated MIB must be semantically equivalent, except where objects or events are omitted because no translation is possible (use of Counter64). Some machine readable information in SMIv2 will be converted into textual descriptions in SMIv1 during the translation process. However, this loss of machine readable information is not considered to change the semantics of the MIB .

\subsection{Structure of the MIB}

The SLAPM-MIB consists of the following components:

o scalar objects

- slapmpolicyNameTable

- slapmpolicyRulestatsTable (equivalent to the deprecated slapmPolicystatsTable)

- slapmPRMonTable (equivalent to the deprecated slapmPolicyMonitorTable)

- slapmSubcomponentTable

Refer to the compliance statement defined within SLAPM-MIB for a definition of what objects and notifications MUST be implemented by all systems as opposed to those that MUST be implemented by end systems only.

Initially most of the tables defined by the MIB module within this document where directly indexed using a policy's name and a subordinate traffic profile name. Over time the structure and resulting naming has grown more complex and as such has exceeded the capacity of being used as a direct MIB table index. As a result of this the original tables (slapmpolicystatsTable and 
slapmpolicyMonitorTable) have been deprecated and replaced with new tables that use an Unsigned32 index element instead of "names". A new table has been defined, slapmpolicyNameTable, that maps the Unsigned32 index to a unique name associated with a given policy rule definition.

\subsection{Scalar objects}

Global objects defined within SLAPM-MIB:

- slapmSpinLock

Enables multiple management application access to SLAPM-MIB. An agent MUST implement the slapmSpinLock object to enable management applications to coordinate their use of the SLAPM-MIB. Management application use of slapmSpinLock is OPTIONAL.

- slapmPolicyCountQueries, slapmPolicyCountAccesses, slapmPolicyCountSuccessAccesses, and slapmPolicyCount NotFounds

Basic statistics on the amount of policy directory access that has occurred at a system.

o slapmPolicypurgetime

Used to prevent the entries in various SLAPM-MIB tables that relate to a policy definition from immediately being deleted when the corresponding policy definition no longer exists. This gives management applications time to discover this condition and close out any polled based interval data that may be being collected. All dependent slapmPRMonTable entries are also deleted when its parent slapmpolicyRulestatsEntry is removed. Refer to the OBJECT description for slapmpolicyPurgetime for a more precise description of this function.

- slapmpolicyTrapEnable

This object enables or suppresses generation of slapmPolicyRuleDeleted or slapmPolicyRuleMonDeleted notifications.

- slapmpolicyTrapFilter

This object enables suppression of slapmSubcMonitorNotokay notifications. 


\section{2 slapmPolicyNameTable}

The slapmpolicyNameTable maps a Unsigned32 index to a unique name associated with a given policy rule definition.

Currently, the core schema definition being worked on within the Policy Framework working group defines five general classes: policyGroup, policyRule, policyCondition, policyTimePeriodCondition, and policyAction. "Policies can either be used in a stand-alone fashion or aggregated into policy groups to perform more elaborate functions. Stand-alone policies are called policy rules. Policy groups are aggregations of policy rules, or aggregations of policy groups, but not both." Each policy rule consists of a set of conditions and a set of actions. Policy rules may be aggregated into policy groups.

"Instances in a directory are identified by distinguished names (DNs), which provide the same type of hierarchical organization that a file system provides in a computer system. A distinguished name is a sequence of relative distinguished names (RDNs), where an RDN provides a unique identifier for an instance within the context of its immediate superior, in the same way that a filename provides a unique identifier for a file within the context of the folder in which it resides."

Each of these instances can also be named to fit in with the existing DEN practice with a commonName (cn) attribute as oppose to the classes name attribute.

"The cn, or commonName, attribute is an X.500 attribute. It stands for commonName. It specifies a user-friendly name by which the object is commonly known. This name may be ambiguous by itself. This name is used in a limited scope (such as an organization). It conforms to the naming conventions of the country or culture with which it is associated. $\mathrm{CN}$ is used universally in DEN as the naming attribute for a class."

An slapmpolicyNameEntry contains a single object, slapmPolicyNameofRule, that contains the unique name associated with a policy rule instance. An slapmPolicyNameEntry is indexed by a Unsigned32 index, slapmPolicyNameIndex, that is assigned by the implementation of this MIB. 


\section{3 slapmPolicyRulestatsTable}

This table is functionally equivalent to the deprecated slapmpolicystatsTable. The slapmPolicystatsTable uses the name of both a policy definition and a traffic profile name to index an entry. The slapmPolicyRulestatsTable uses an slapmPolicyNameEntry index (Unsigned32) instead.

The slapmPolicyRulestatsTable is the main table defined by SLAPM-MIB. The primary index for this table is slapmpolicyNamesystemAddress that enables support of multiple systems from a single policy agent. The index element, slapmpolicyNamesystemAddress, value must be either the zero-length octet string when at a policy agent only a single system is being support, 4 octets for a ipv4 address, or 16 octets for a ipv6 address.

It is possible that on a single system multiple policy agent instances exists. The Entity MIB, refer to [19], should be used to handle the resulting MIBs.

With respect to slapmPolicyNameSystemAddress one slapmpolicyRulestatsEntry exists for each policy rule instance. Entries in this table are not administered via SNMP. An agent implementation for this table MUST reflect its current set of policy rule instances via table entries. The mechanisms for policy administration are outside of the scope of this memo.

\section{4 slapmPRMonTable}

This table is functionally equivalent to the deprecated slapmPolicyMonitorTable. The slapmPolicyMonitorTable uses the name of both a policy definition and a traffic profile name to index an entry. The slapmPRMonTable uses an slapmPolicyNameEntry index (Unsigned32) instead.

The slapmPRMonTable provides a method of monitoring the effect of SLA policy being used at a system. A management application creates an slapmPRMonEntry for each collection that it requires. The value of the BITS slapmPRMonControl object determines what type of monitoring occurs, at what level to monitor and whether trap support is enabled:

- monitorMinRate (0)

Use the value of slapmPRMonInterval as the interval to determine current traffic in and out rates, using slapmPRMoncurrentInRate and slapmPRMonCurrentoutRate, that can be compared to slapmPRMonMinRateLow for determining when to generate a slapmPolicyRuleMonNotokay notification. The notification 
slapmPolicyRuleMonokay is generated when the problem is resolved. This can be determined by comparing the current rates to slapmPRMonMinRateHigh.

o monitorMaxRate (1)

Use the value of slapmPRMonInterval as the interval to determine current traffic in and out rate, using slapmPRMoncurrentInRate and slapmPRMonCurrentoutRate, that can be compared to slapmPRMonMaxRateHigh for determining when to generate a slapmPolicyRuleMonNotokay notification. The notification slapmPolicyRuleMonokay is generated when the problem is resolved. This can be determined by comparing the current rates to slapmPRMonMaxRateLow.

- monitorMaxDelay (2)

Use the value of slapmPRMonInterval as the interval to determine the current delay. This can be calculated on an aggregate level by averaging the round trip times for all TCP connections associated with the policy definition. For an individual subcomponent its round trip time can be used directly. Compare this value to slapmPRMonMaxDelayHigh for determining when to generate a slapmPolicyRuleMonNotokay notification. The notification slapmPolicyRuleMonokay is generated when the problem is resolved. This can be determined by comparing the current rates to slapmPRMonMaxDelayLow.

UDP subcomponents don't support max delay monitoring .

- enableAggregateTraps (3)

The slapmPRMonitorControl BITS setting, enableAggregateTraps(3), MUST be set in order for any notifications relating to slapmpolicyRulestatsTable monitoring to be generated.

- enableSubcomponentTraps (4)

This slapmPRMonControl BITS setting MUST be set in order for any notifications relating to slapmSubcomponetTable monitoring to be generated. The slapmPRMonControl BITS setting monitorSubcomponents(5) MUST be selected in order for this setting to be allowed.

- monitorSubcomponents (5)

If selected monitor slapmSubcomponentTable entries individually. Note: aggregate policy rule monitoring is always enabled. 
The index element slapmPRMonOwnerIndex is used as the first index in slapmPRMonTable in order to enable SNMP VACM security control. The slapmPRMonTable is the only table that supports SNMP Rowstatus operations.

\section{5 slapmSubcomponentTable}

Entries are made into this table for the protocol entities (policy traffic profile subcomponents) to indicate actual policy rule usage, provide general statistics at either a TCP connection or UDP listener level, and enable subcomponent monitoring.

\subsection{Definitions}

SLAPM-MIB DEFINITIONS : := BEGIN

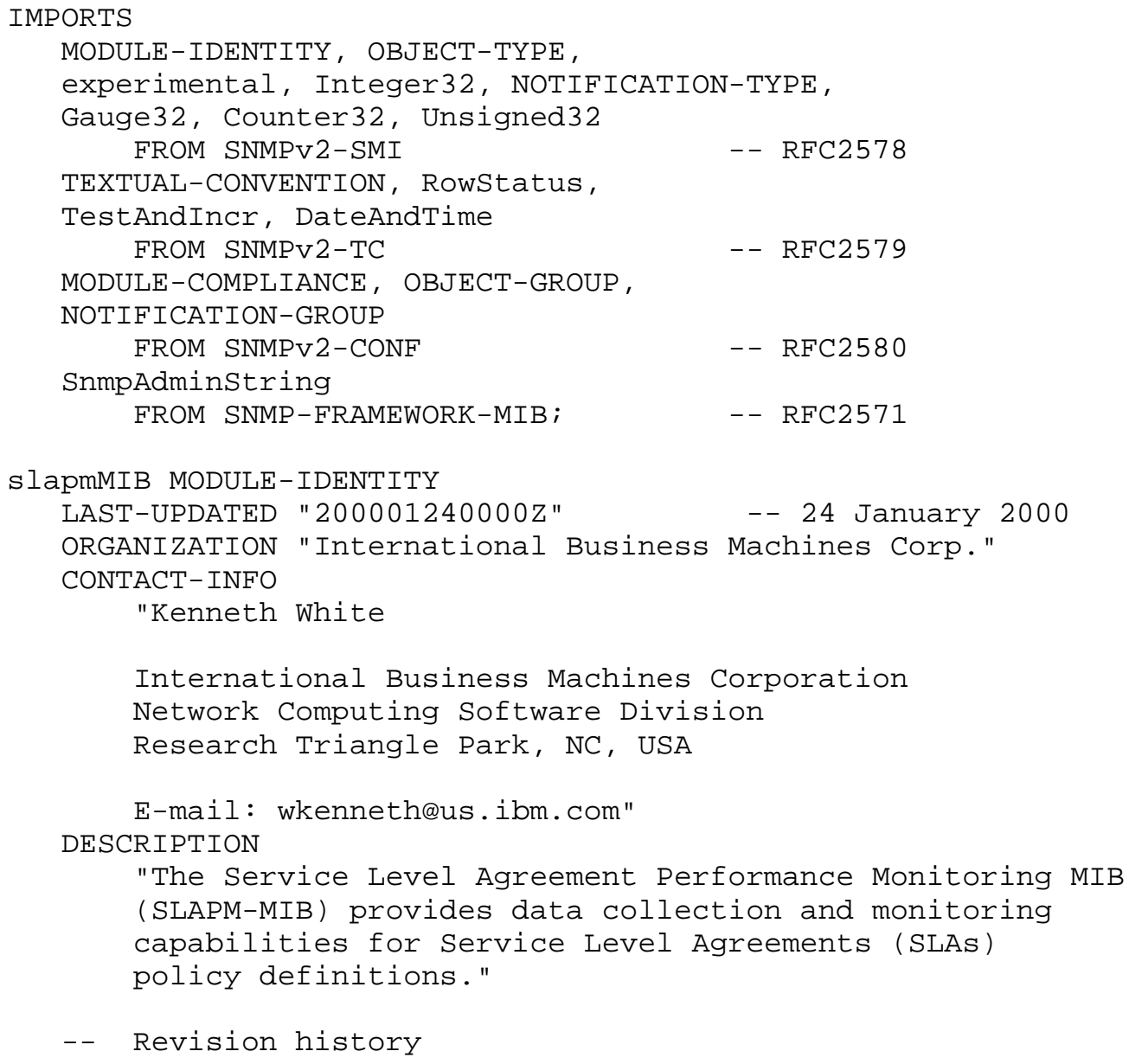




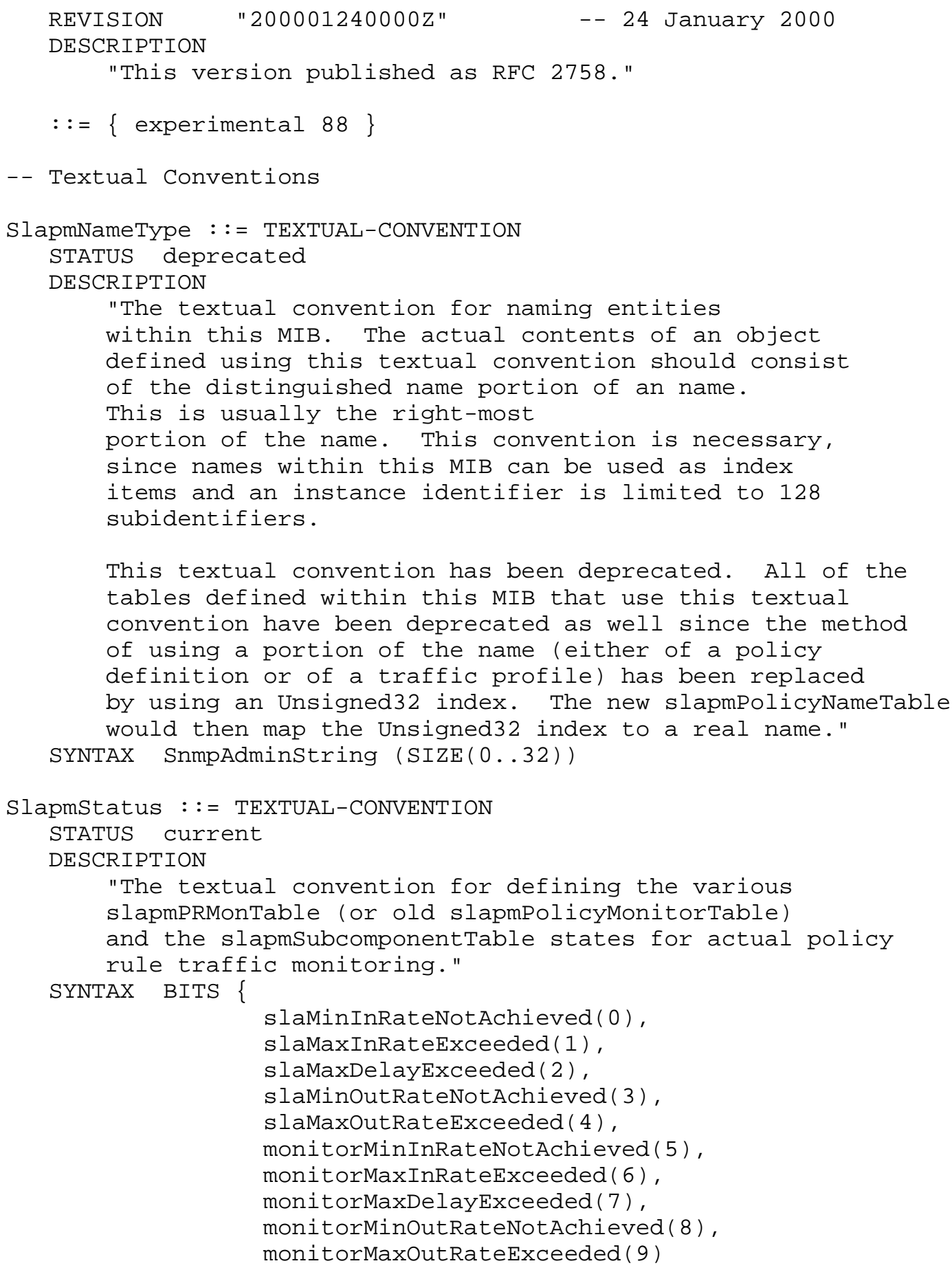




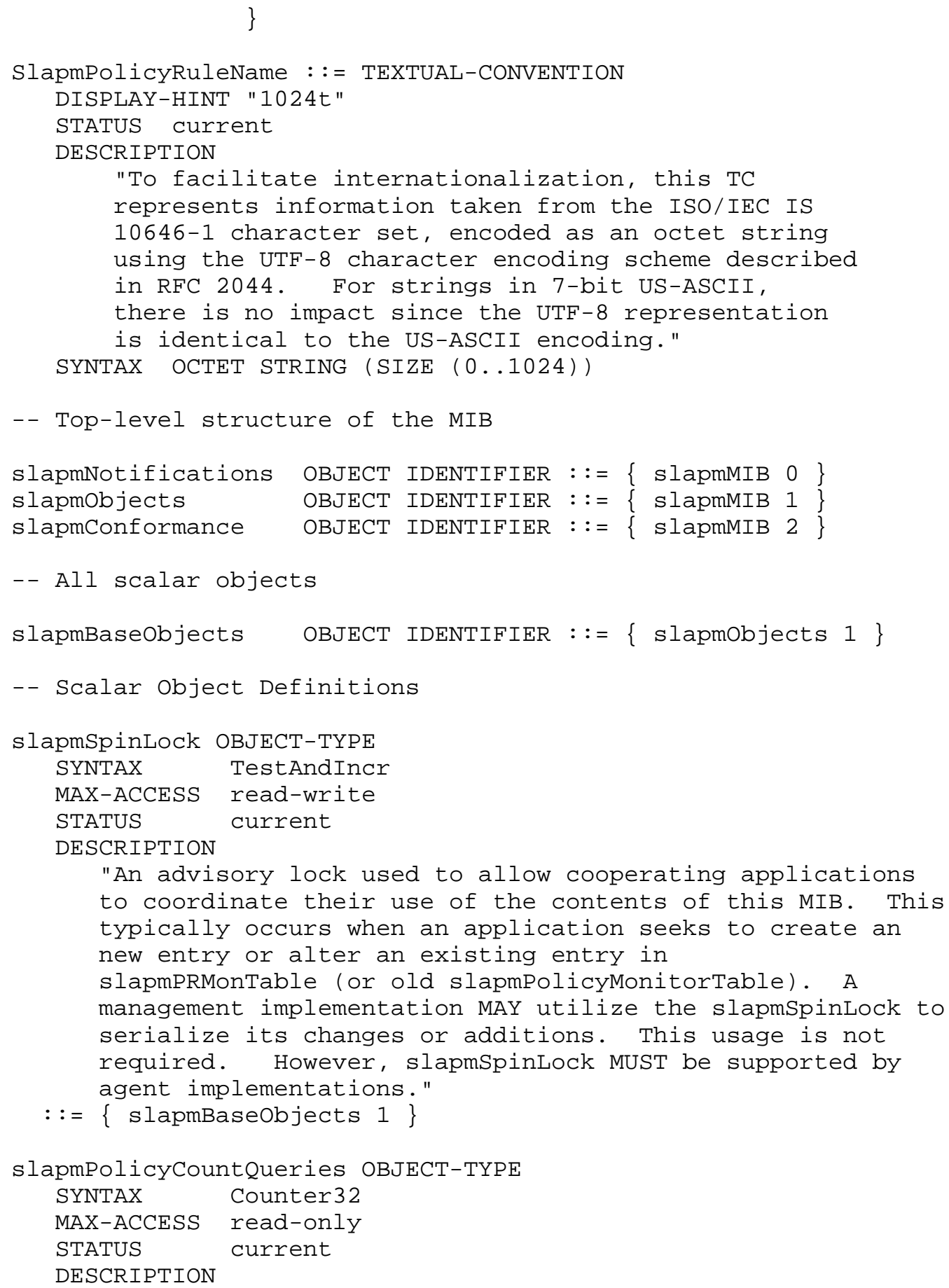




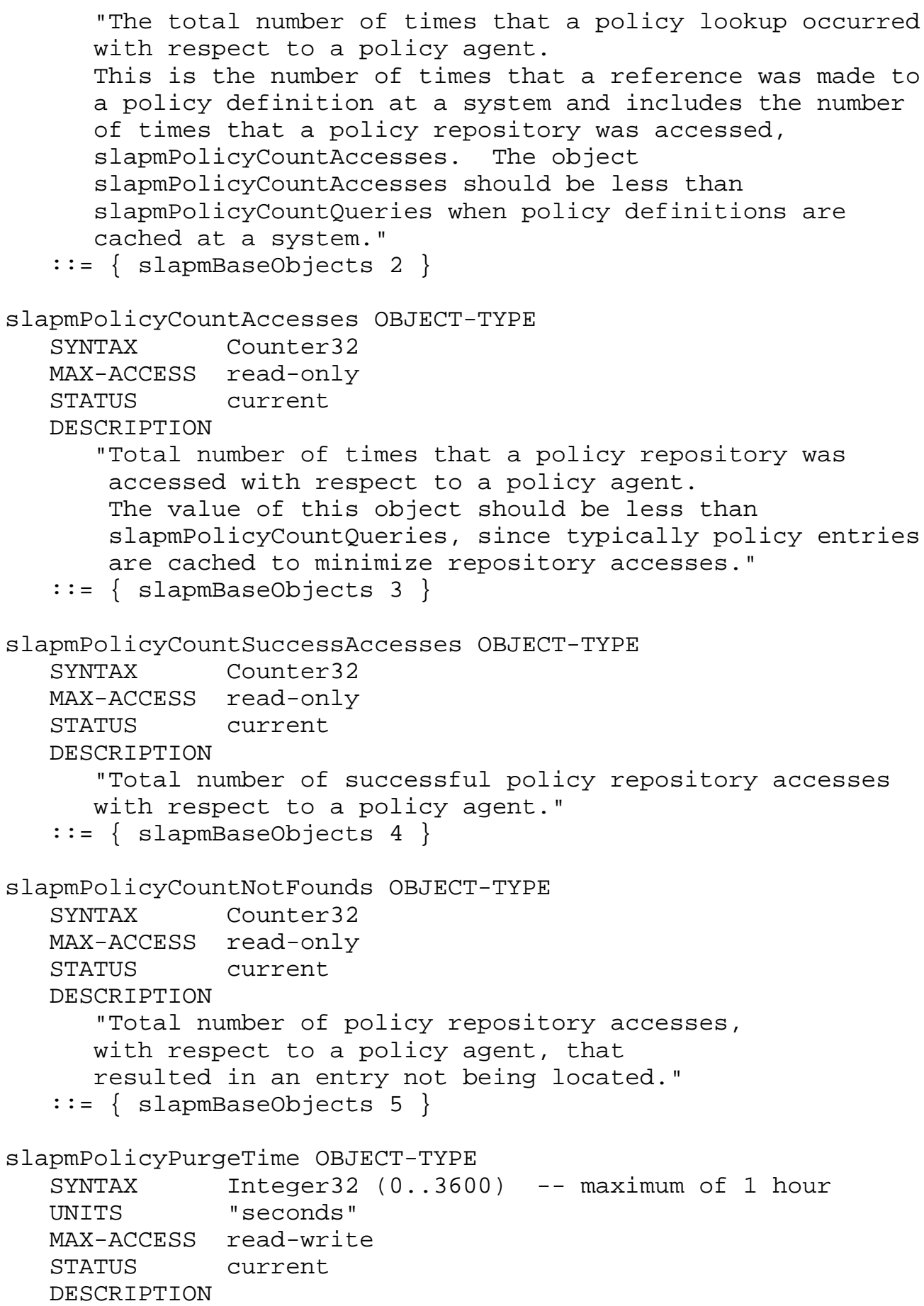




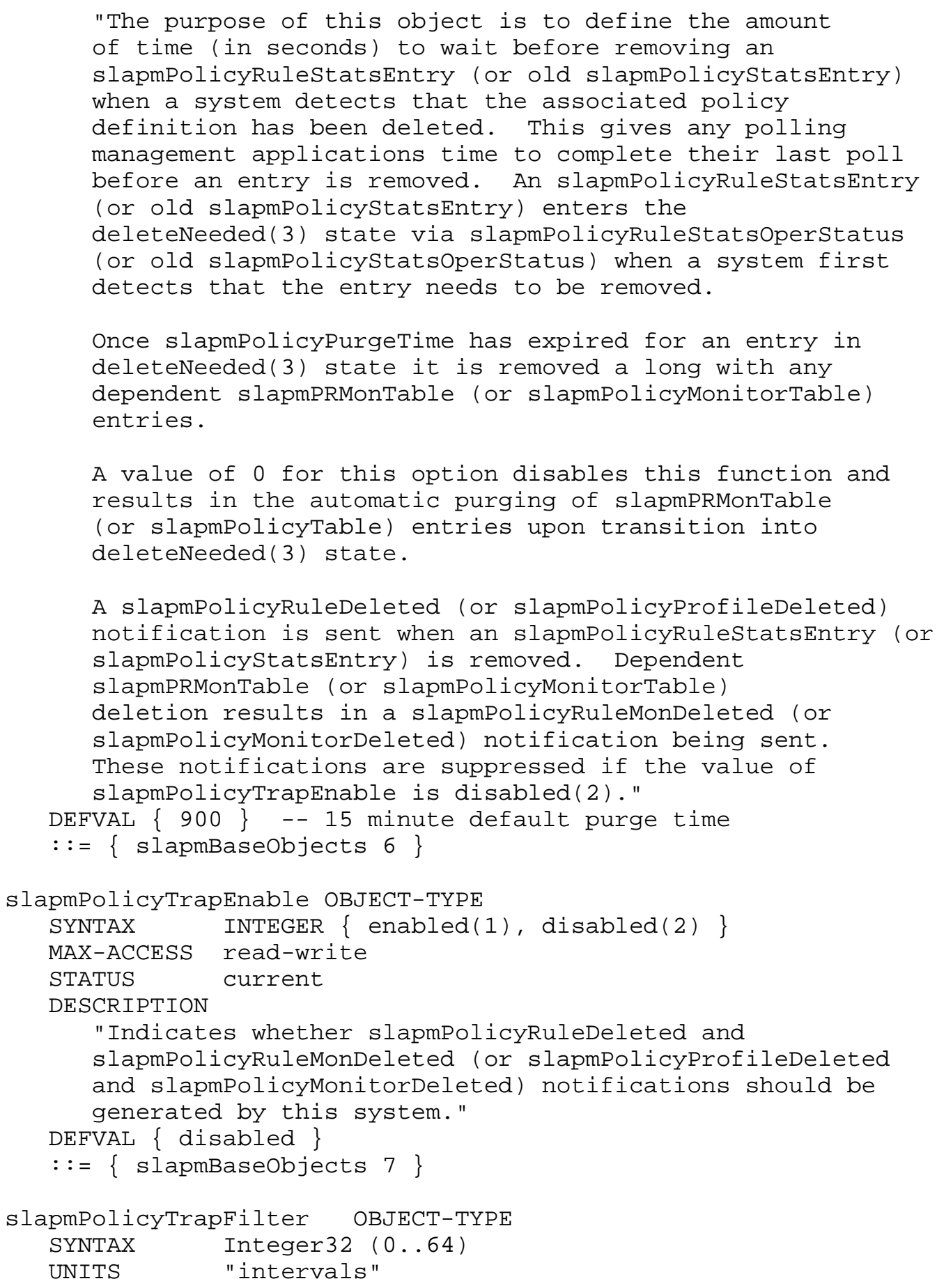




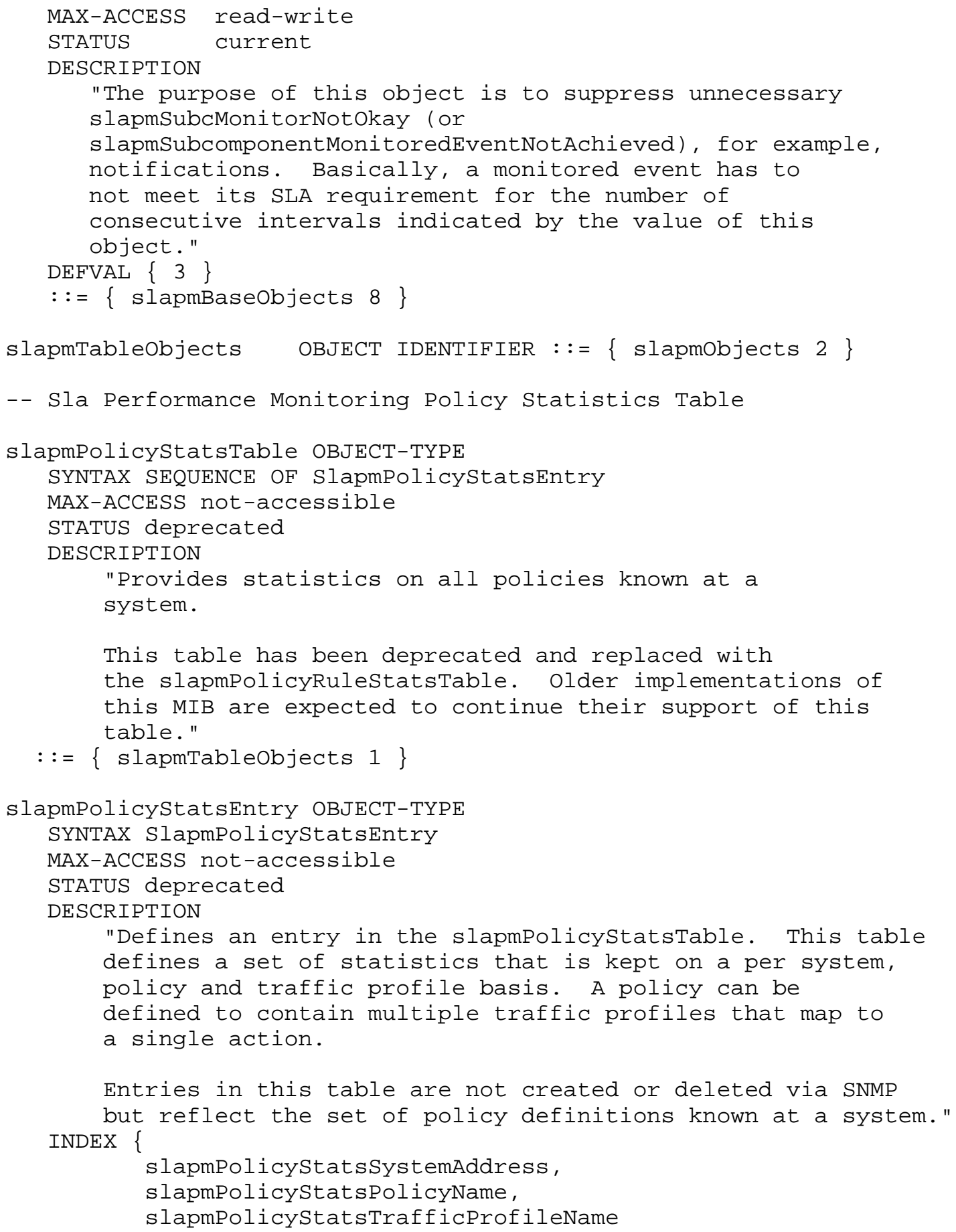


$::=\left\{\begin{array}{l}\} \\ \text { slapmPolicyStatsTable } 1\end{array}\right\}$

SlapmPolicystatsEntry : := SEQUENCE \{

slapmPolicystatsSystemAddress OCTET STRING, slapmPolicystatsPolicyName slapmPolicystatsTrafficProfileName slapmPolicystatsoperstatus slapmPolicystatsActiveconns slapmPolicystatsTotalconns slapmPolicystatsFirstActivated slapmPolicystatsLastMapping slapmPolicystatsInoctets slapmPolicystatsoutoctets slapmPolicystatsConnectionLimit slapmPolicyStatsCountAccepts slapmPolicystatsCountDenies slapmPolicystats InDiscards slapmPolicystatsOutDiscards slapmPolicystatsInPackets slapmPolicyStatsOutPackets slapmPolicystats InProfileoctets slapmPolicystatsoutProfileoctets slapmPolicystatsMinRate slapmPolicystatsMaxRate 


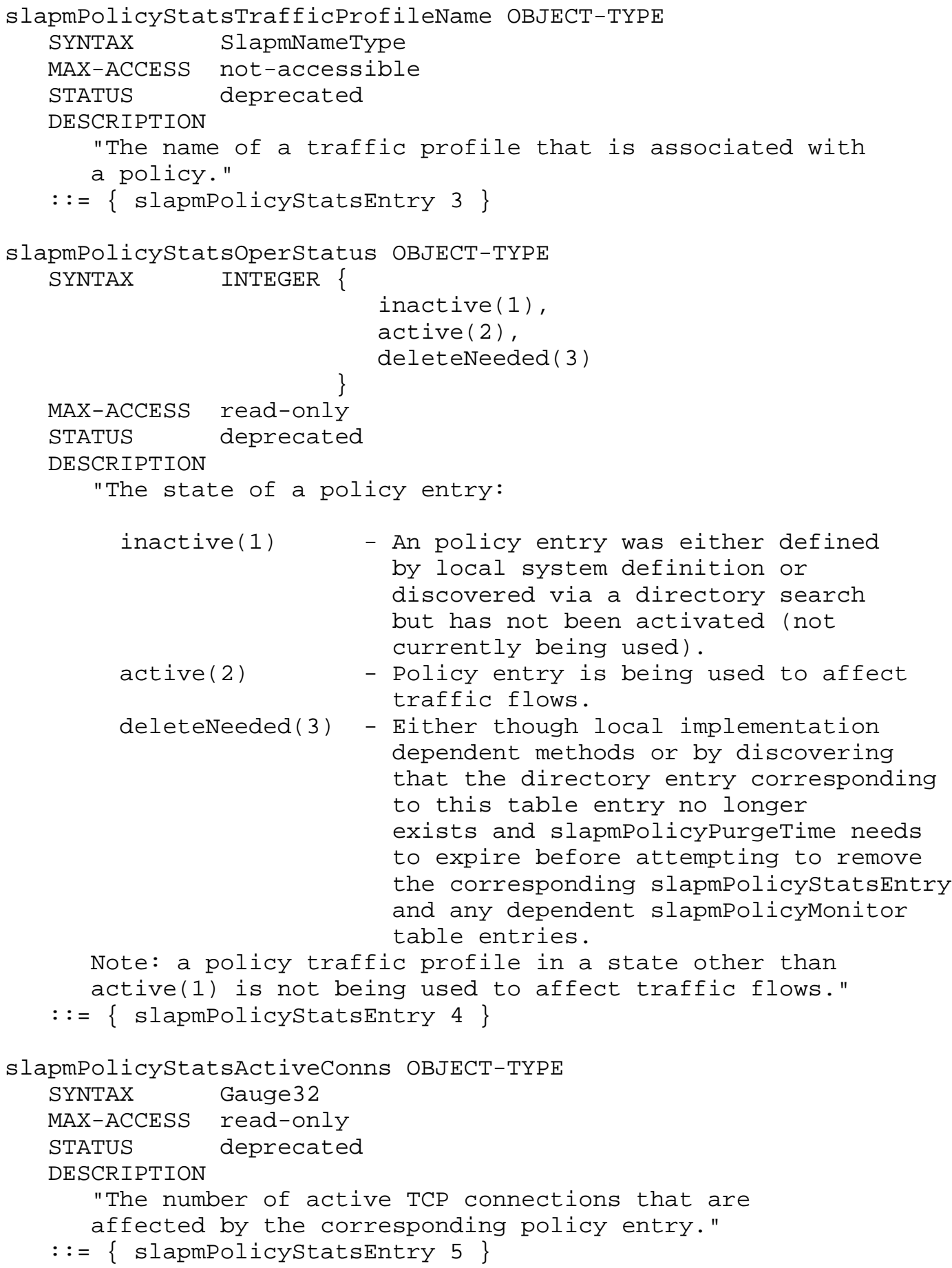




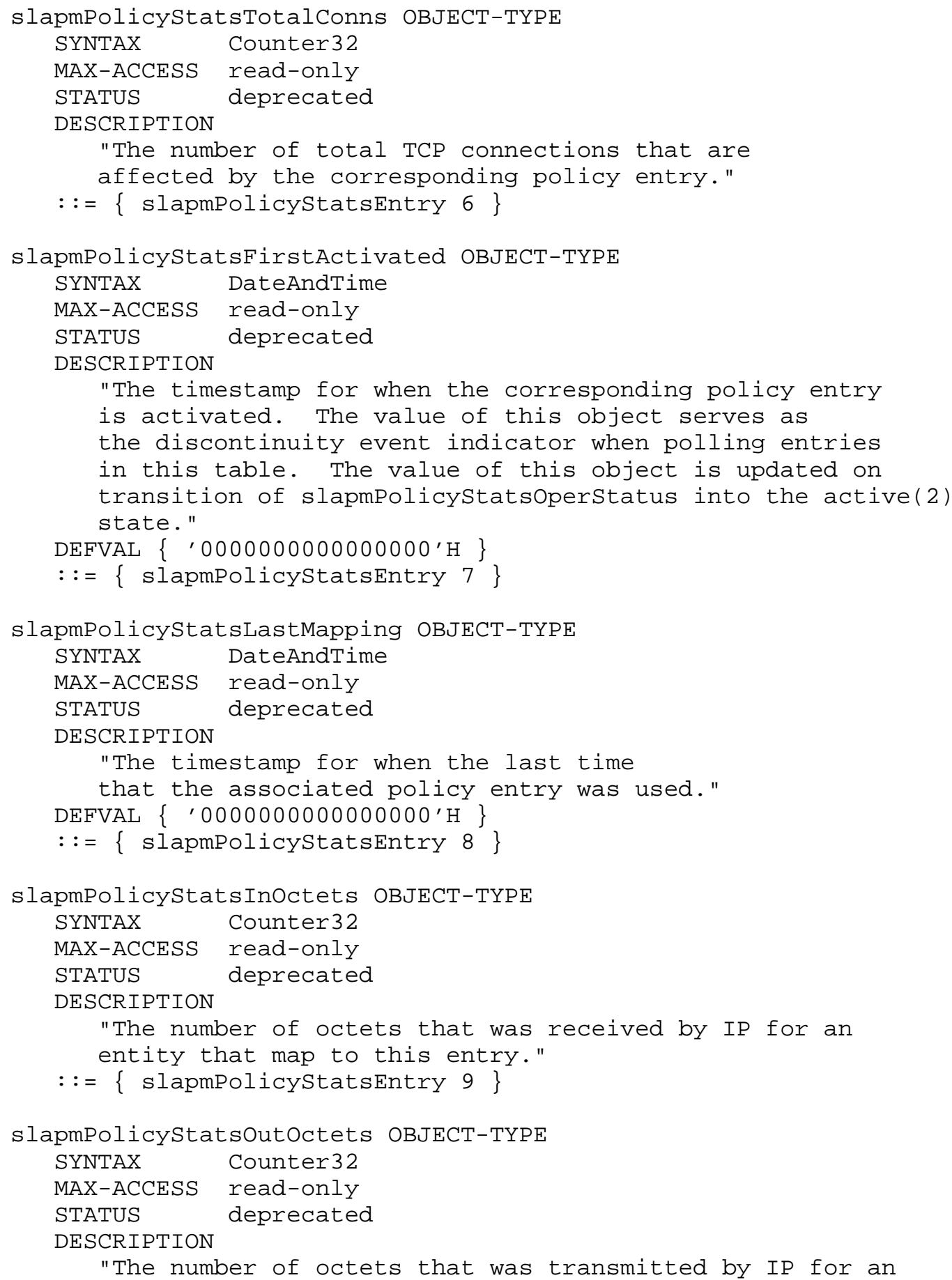




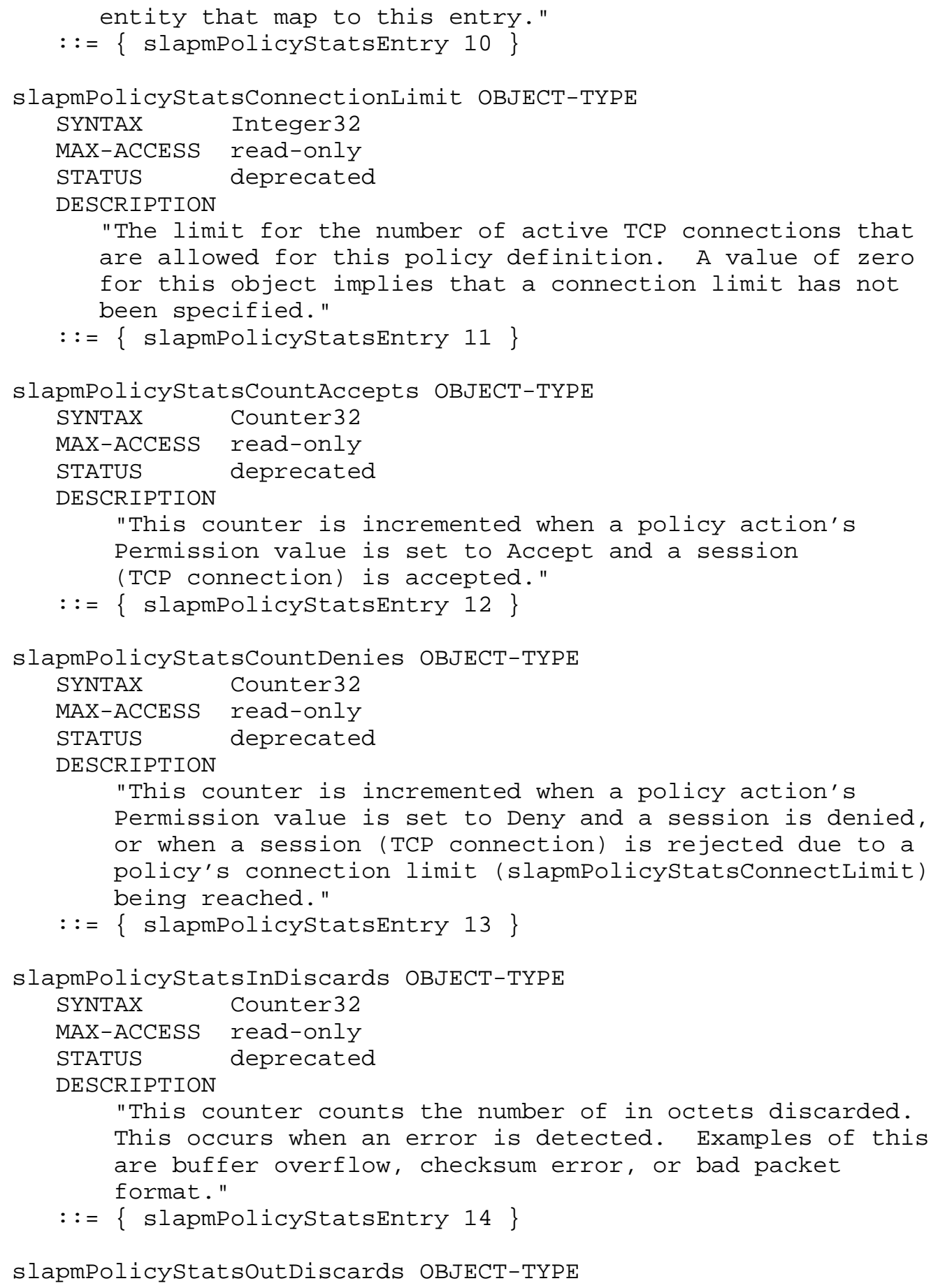




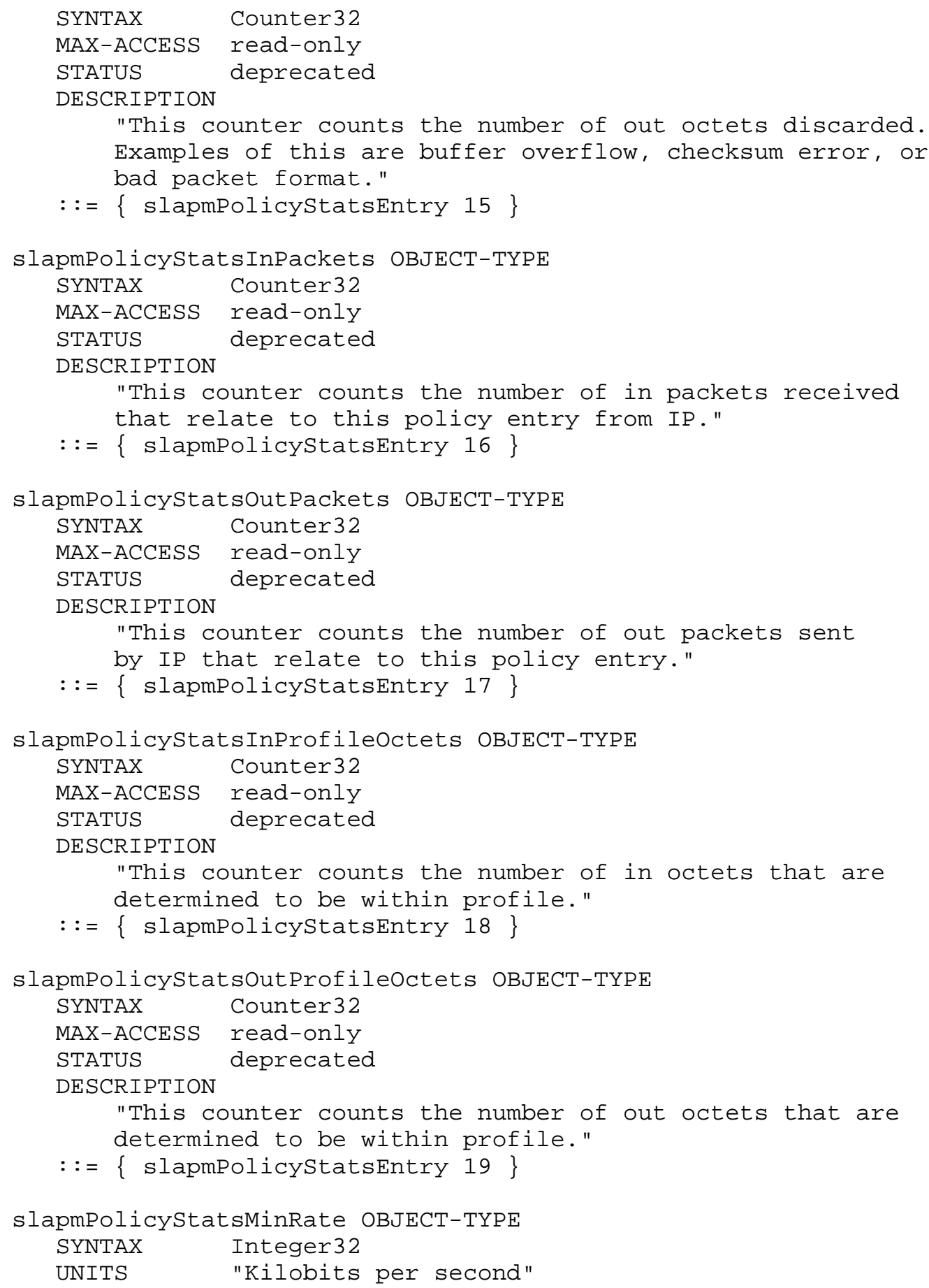




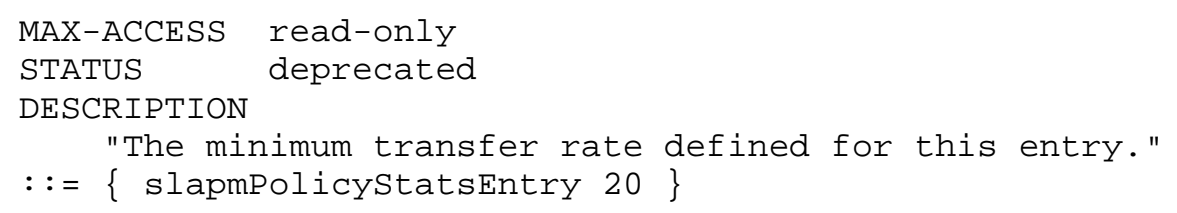




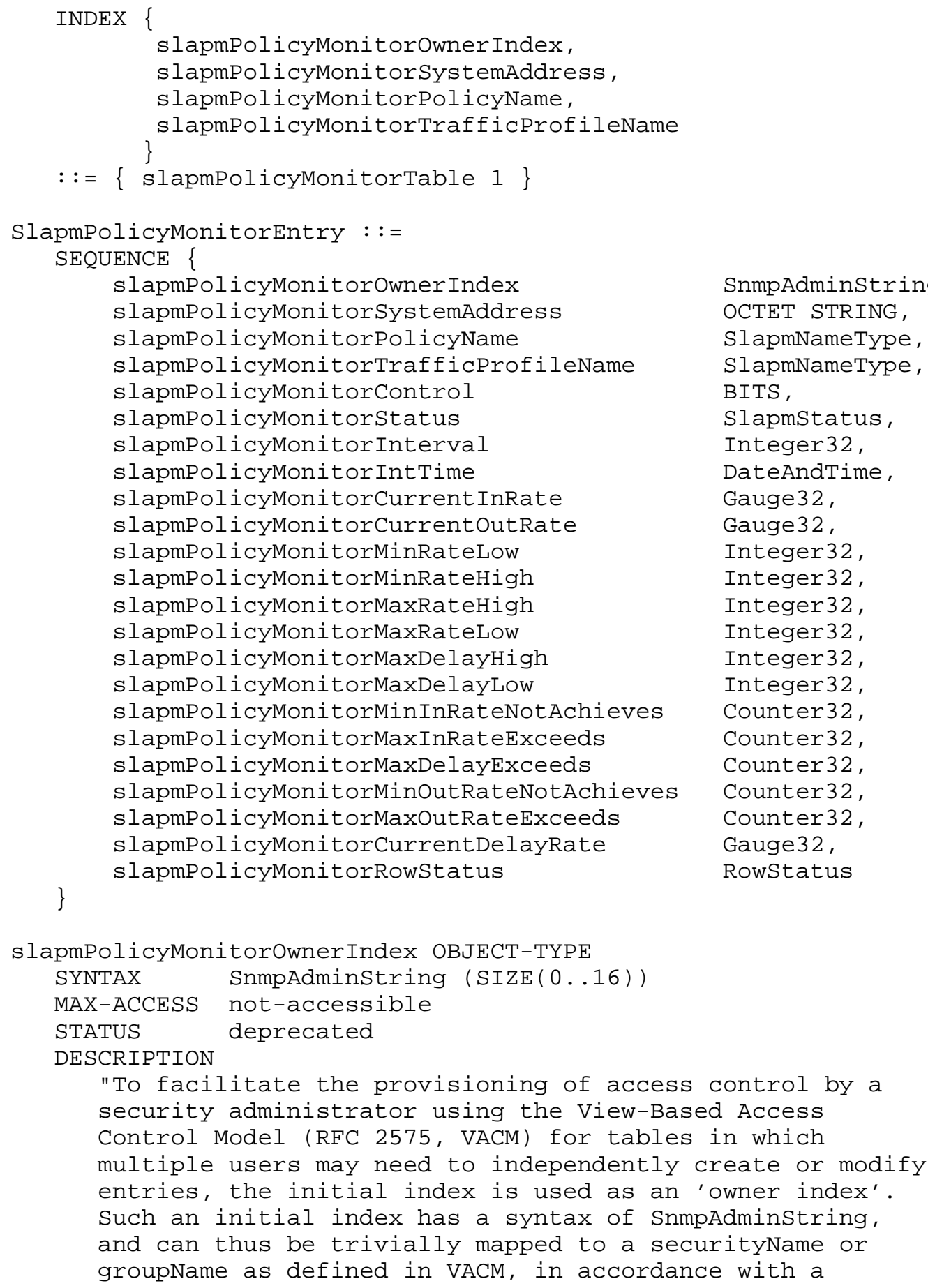




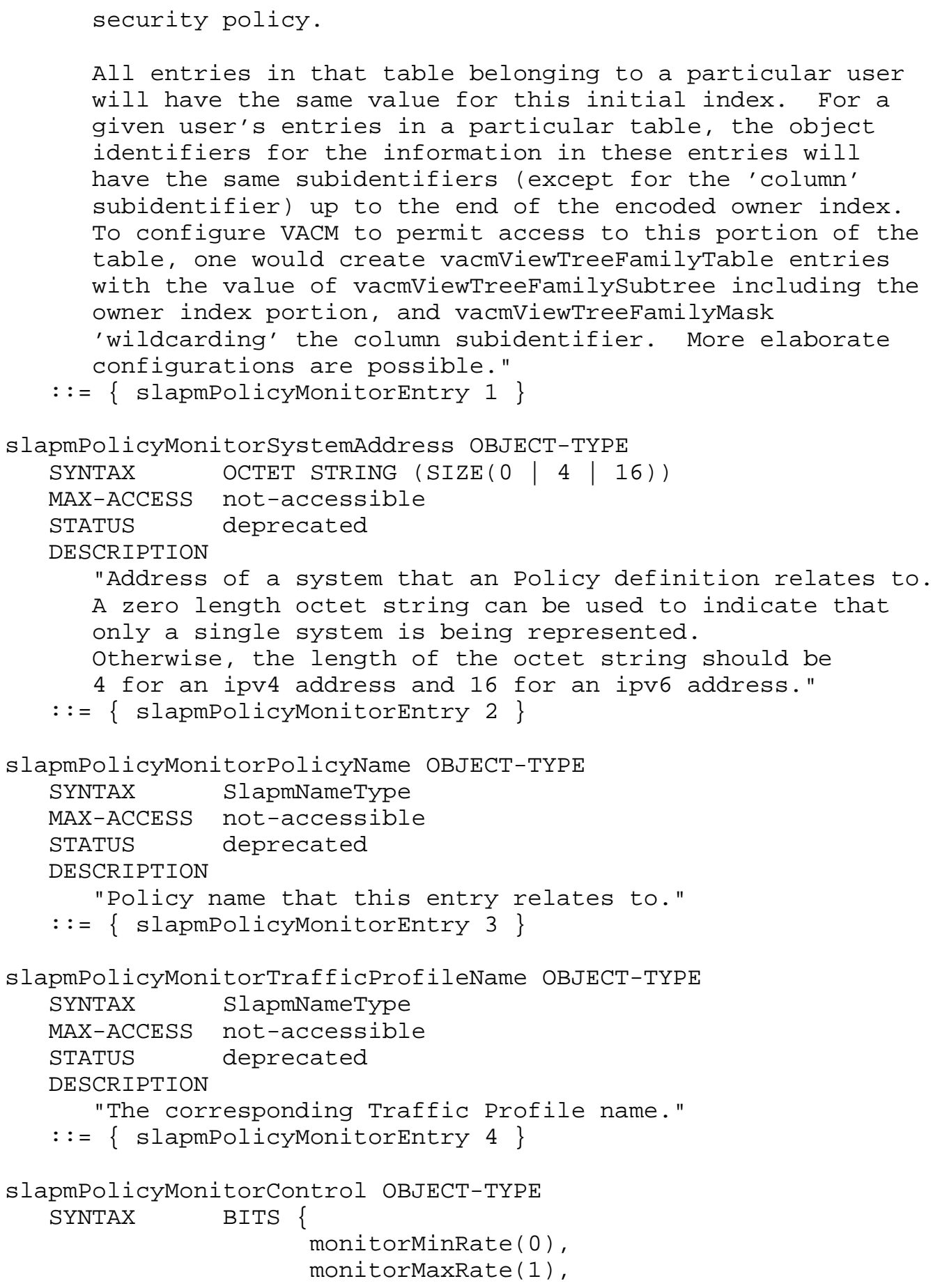




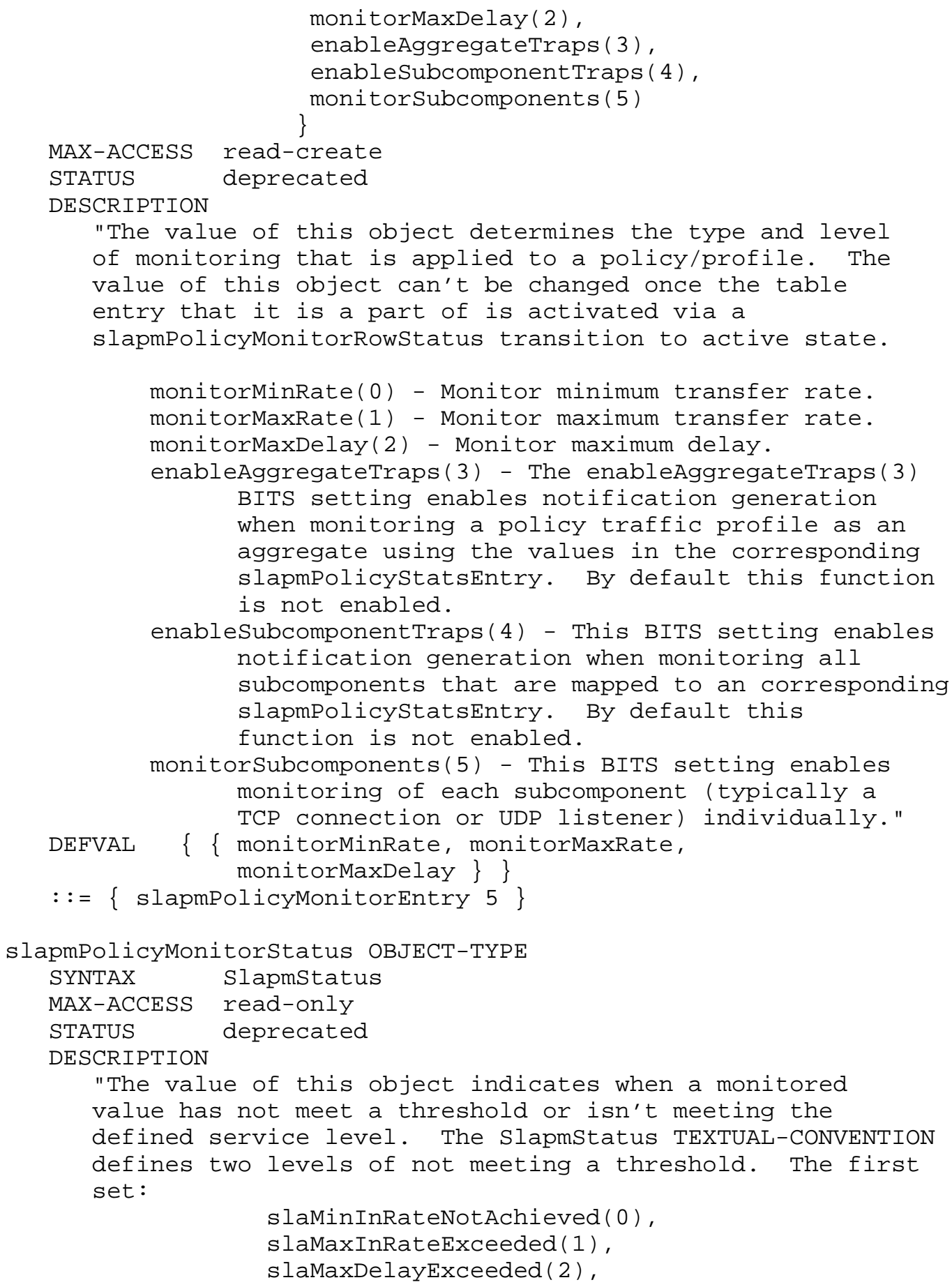




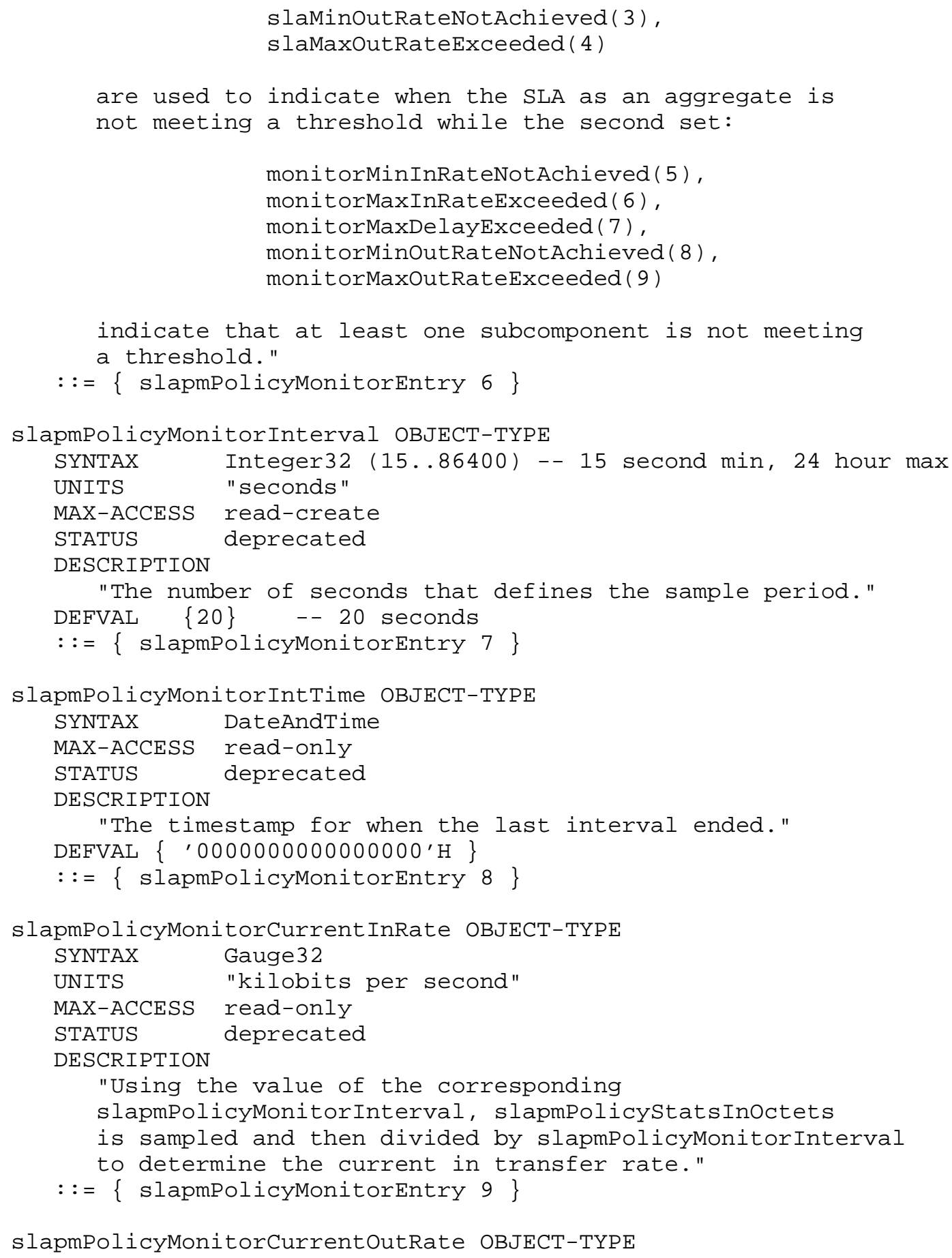




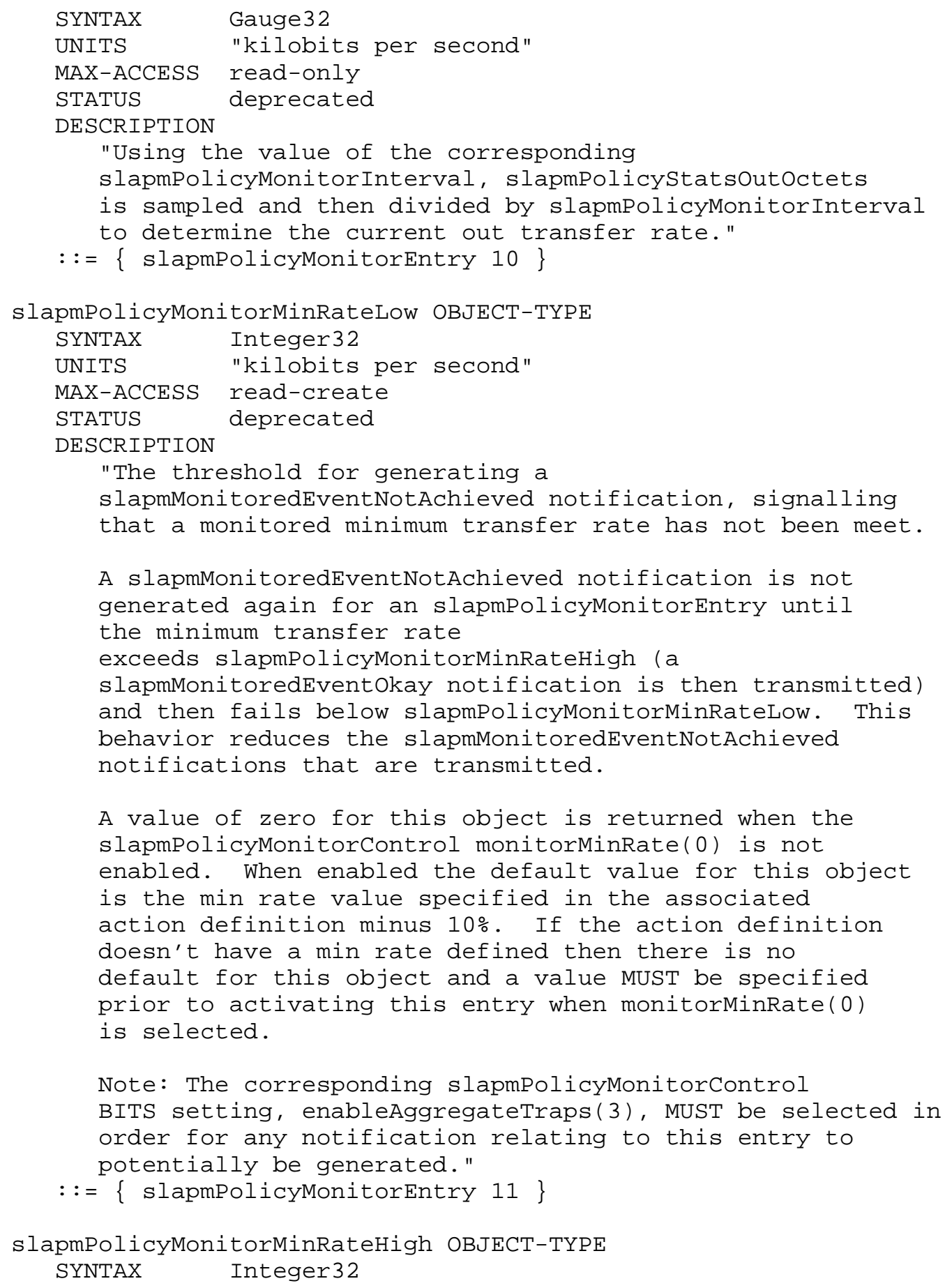




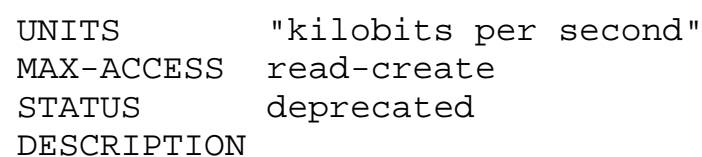

"The threshold for generating a slapmMonitoredEventokay notification, signalling that a monitored minimum transfer rate has increased to an acceptable level.

A value of zero for this object is returned when the slapmPolicyMonitorControl monitorMinRate(0) is not enabled. When enabled the default value for this object is the min rate value specified in the associated action definition plus 10\%. If the action definition doesn't have a min rate defined then there is no default for this object and a value MUST be specified prior to activating this entry when monitorMinRate(0) is selected.

Note: The corresponding slapmPolicyMonitorControl BITS setting, enableAggregateTraps(3), MUST be selected in order for any notification relating to this entry to potentially be generated."

$::=\{$ slapmPolicyMonitorEntry 12$\}$

slapmPolicyMonitorMaxRateHigh OBJECT-TYPE

SYNTAX Integer32

UNITS "kilobits per second"

MAX-ACCESS read-create

STATUS deprecated

DESCRIPTION

"The threshold for generating a

slapmMonitoredEventNotAchieved notification, signalling

that a monitored maximum transfer rate has been exceeded.

A slapmMonitoredEventNotAchieved notification is not generated again for an slapmPolicyMonitorEntry until the maximum transfer rate fails below

slapmPolicyMonitorMaxRateLow (a slapmMonitoredEventokay notification is then transmitted) and then raises above slapmPolicyMonitorMaxRateHigh. This behavior reduces the slapmMonitoredEventNotAchieved notifications that are transmitted.

A value of zero for this object is returned when the slapmPolicyMonitorControl monitorMaxRate(1) is not enabled. When enabled the default value for this object is the max rate value specified in the associated action definition plus 10\%. If the action definition 


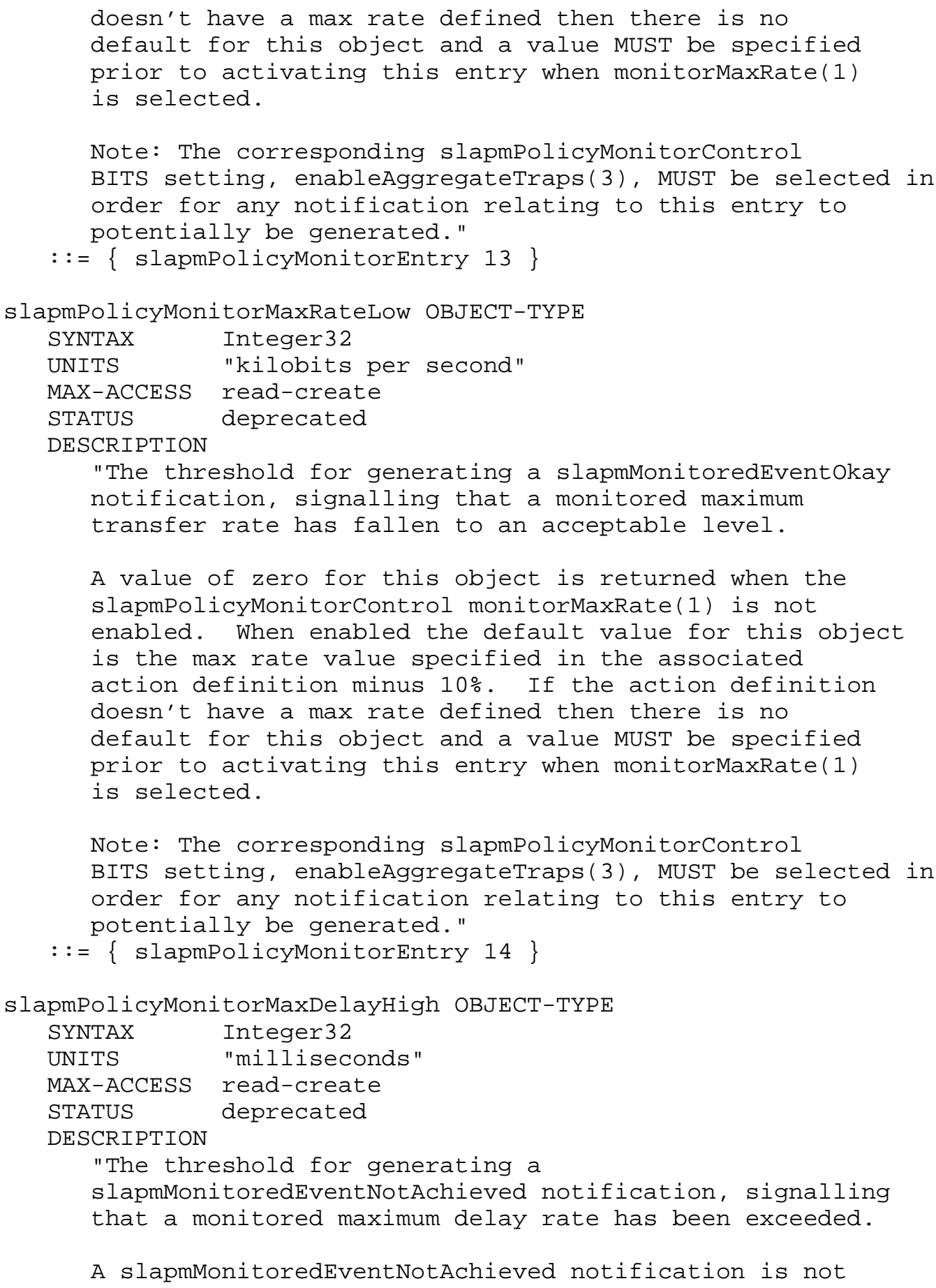


generated again for an slapmPolicyMonitorEntry until

the maximum delay rate falls below

slapmPolicyMonitorMaxDelayLow (a slapmMonitoredEventokay notification is then transmitted) and raises above slapmPolicyMonitorMaxDelayHigh. This behavior reduces the slapmMonitoredEventNotAchieved notifications that are transmitted.

A value of zero for this object is returned when the slapmPolicyMonitorControl monitorMaxDelay(4) is not enabled. When enabled the default value for this object is the max delay value specified in the associated action definition plus $10 \%$. If the action definition doesn't have a max delay defined then there is no default for this object and a value MUST be specified prior to activating this entry when monitorMaxDelay(4) is selected.

Note: The corresponding slapmPolicyMonitorControl BITS setting, enableAggregateTraps(3), MUST be selected in order for any notification relating to this entry to potentially be generated."

$::=\{$ slapmPolicyMonitorEntry 15$\}$

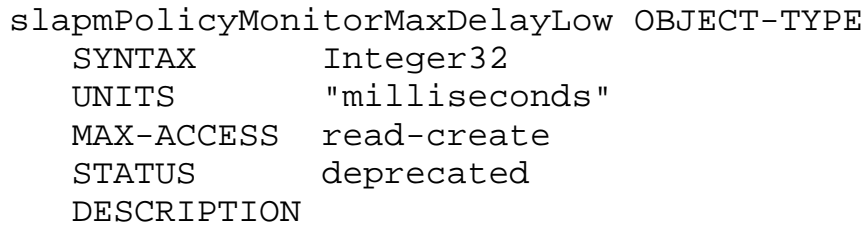

"The threshold for generating a slapmMonitoredEventokay notification, signalling that a monitored maximum delay rate has fallen to an acceptable level.

A value of zero for this object is returned when the slapmPolicyMonitorControl monitorMaxDelay(4) is not enabled. When enabled the default value for this object is the max delay value specified in the associated action definition minus $10 \%$. If the action definition doesn't have a max delay defined then there is no default for this object and a value MUST be specified prior to activating this entry when monitorMaxDelay(4) is selected.

Note: The corresponding slapmPolicyMonitorControl BITS setting, enableAggregateTraps(3), MUST be selected in order for any notification relating to this entry to potentially be generated." 


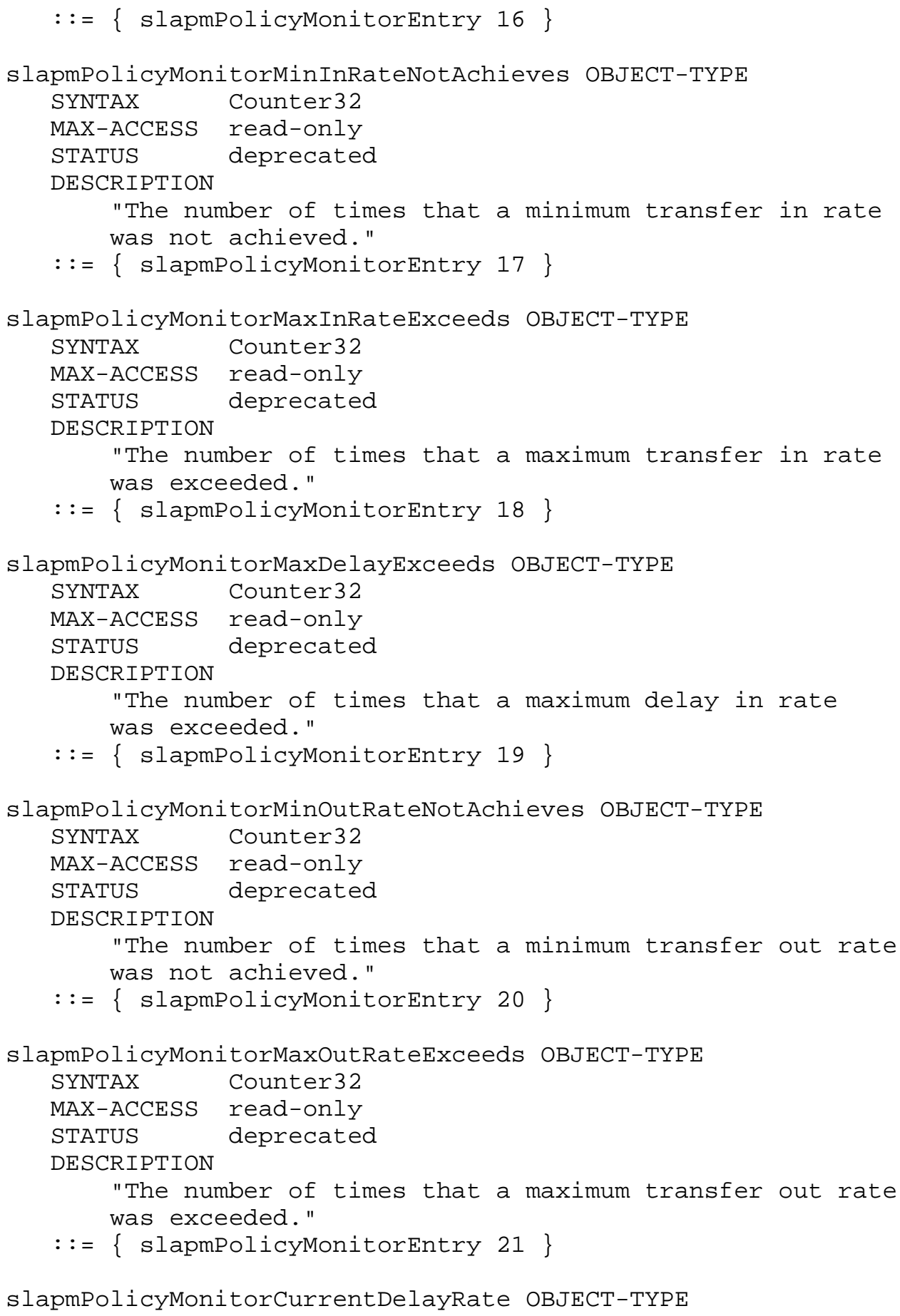




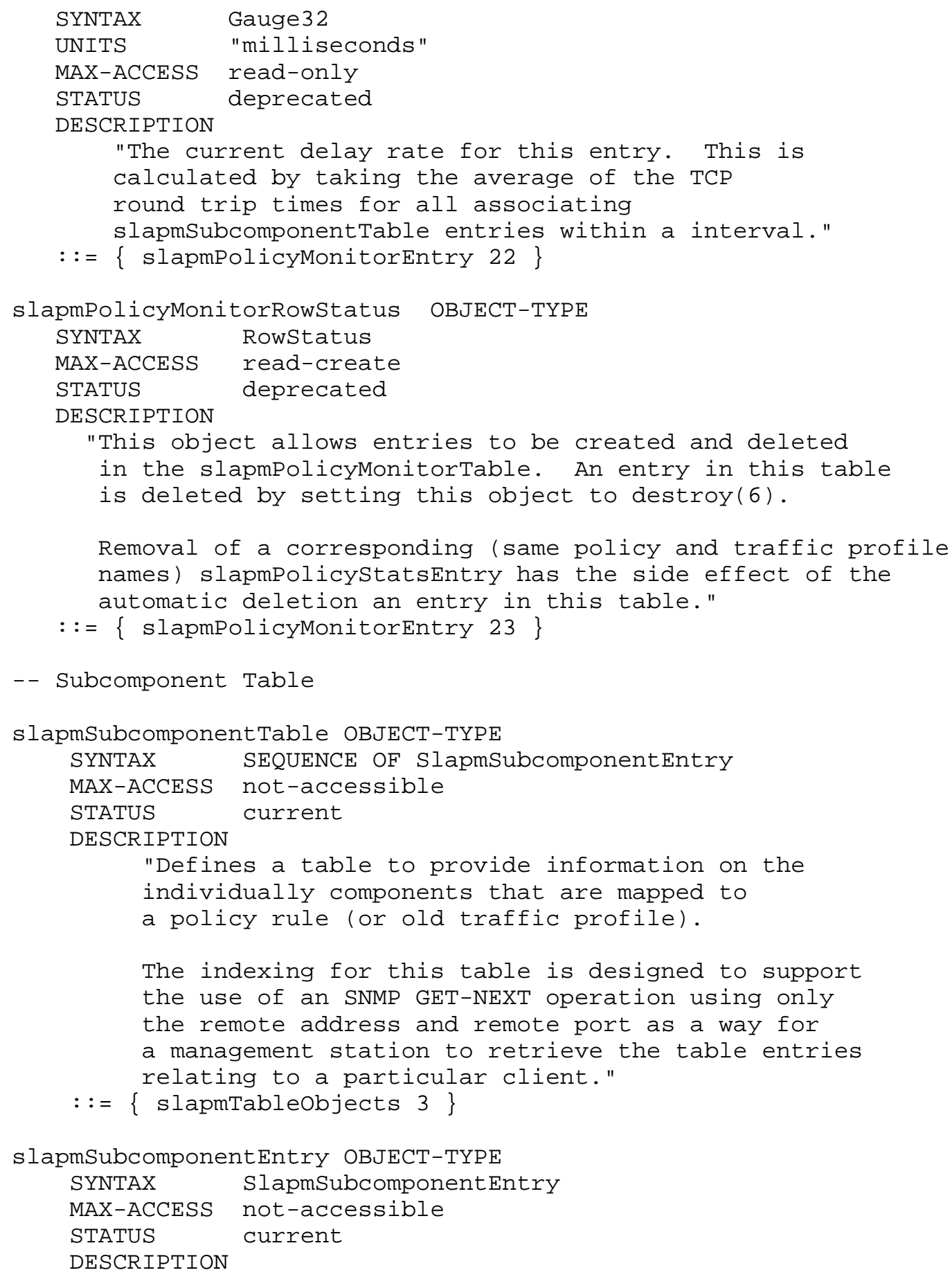


INDEX

"Describes a particular subcomponent entry. This

table does not have an OwnerIndex as

part of its indexing since this table's contents

is intended to span multiple users."

slapmSubcomponentRemAddress, slapmSubcomponentRemPort, slapmSubcomponentLocalAddress, \} slapmSubcomponent LocalPort

$::=\{$ slapmSubcomponentTable 1$\}$

SlapmSubcomponentEntry : := SEQUENCE \{

slapmSubcomponentRemAddress

slapmSubcomponentRemPort

slapmSubcomponent LocalAddress

slapmSubcomponent LocalPort

slapmSubcomponentProtocol

slapmSubcomponent SystemAddress

slapmSubcomponentPolicyName

slapmSubcomponentTrafficProfileName

slapmSubcomponentLastActivity

slapmSubcomponent Inoctets

slapmSubcomponentoutoctets

slapmSubcomponent TcpoutBufferedOctets

slapmSubcomponent TcpInBufferedoctets

slapmSubcomponent TcpReXmts

OCTET STRING, Integer32, OCTET STRING, Integer32, INTEGER, OCTET STRING, SlapmNameType, SlapmNameType, DateAndTime, Counter32, Counter32, Counter32, Counter32, Counter32, slapmSubcomponent TcpRoundTripTime Integer 32 , slapmSubcomponent TcpRoundTripVariance Integer32, slapmSubcomponent InPdus slapmSubcomponentoutPdus Counter32, Counter32, slapmSubcomponentApplName SnmpAdminString, slapmSubcomponentMonitorStatus slapmSubcomponentMonitorIntTime Slapmstatus, slapmSubcomponentMonitorCurrentInRate Gauge32, slapmSubcomponentMonitorCurrentoutRate Gauge32, slapmSubcomponentPolicyRuleIndex Unsigned32

\}

slapmSubcomponentRemAddress OBJECT-TYPE

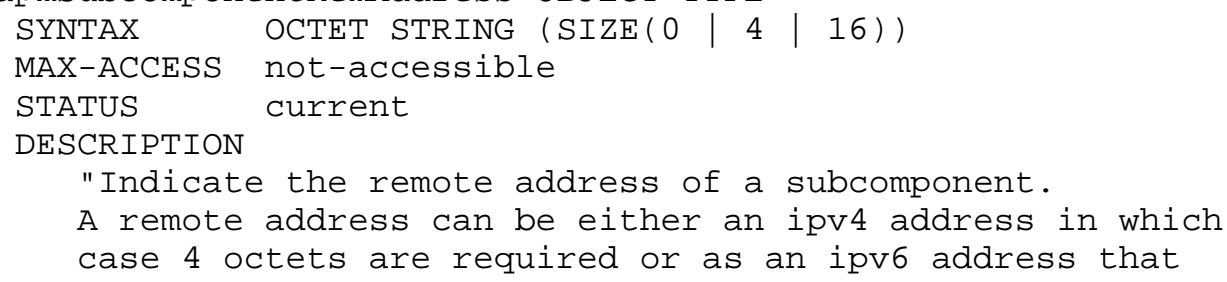




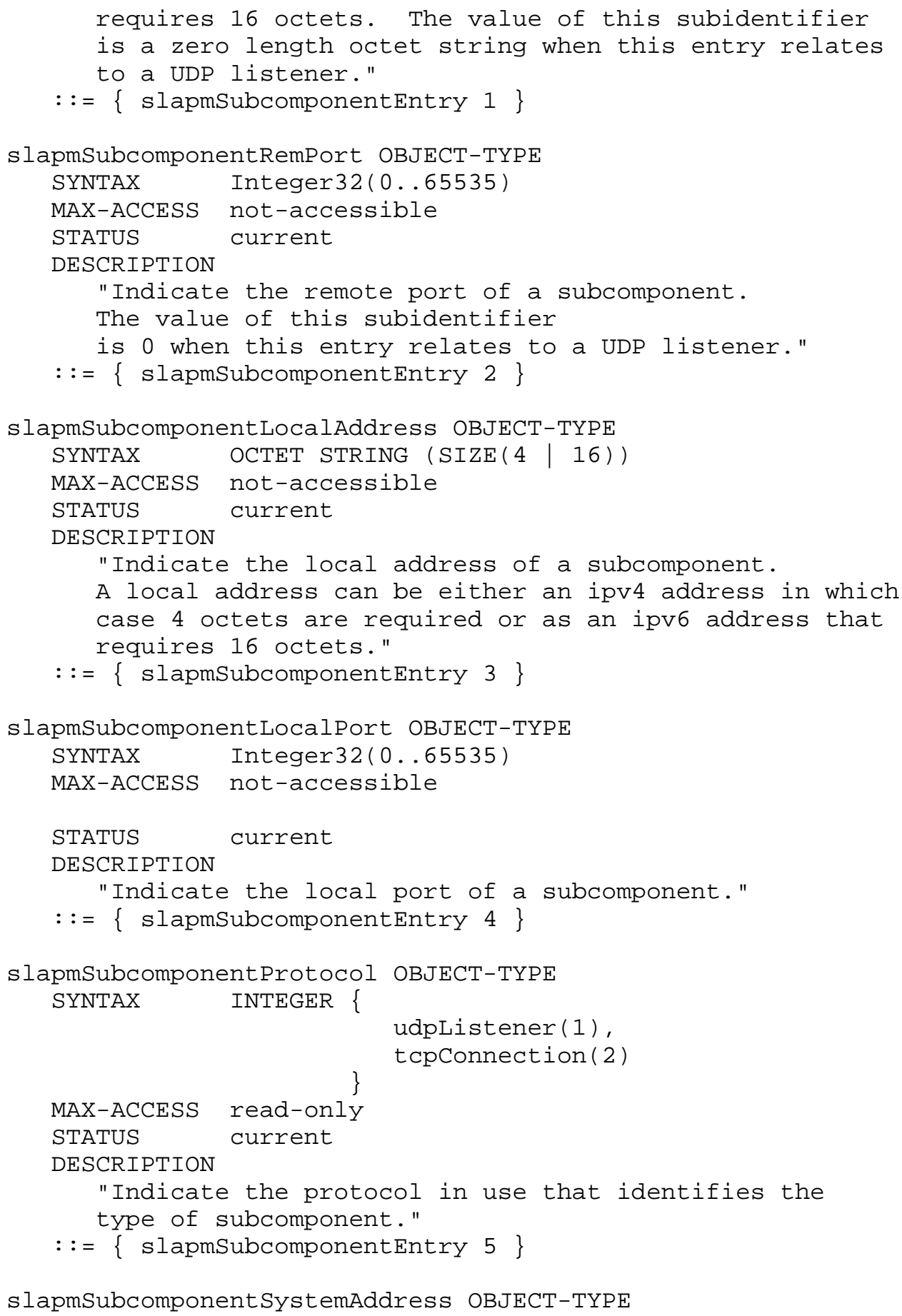




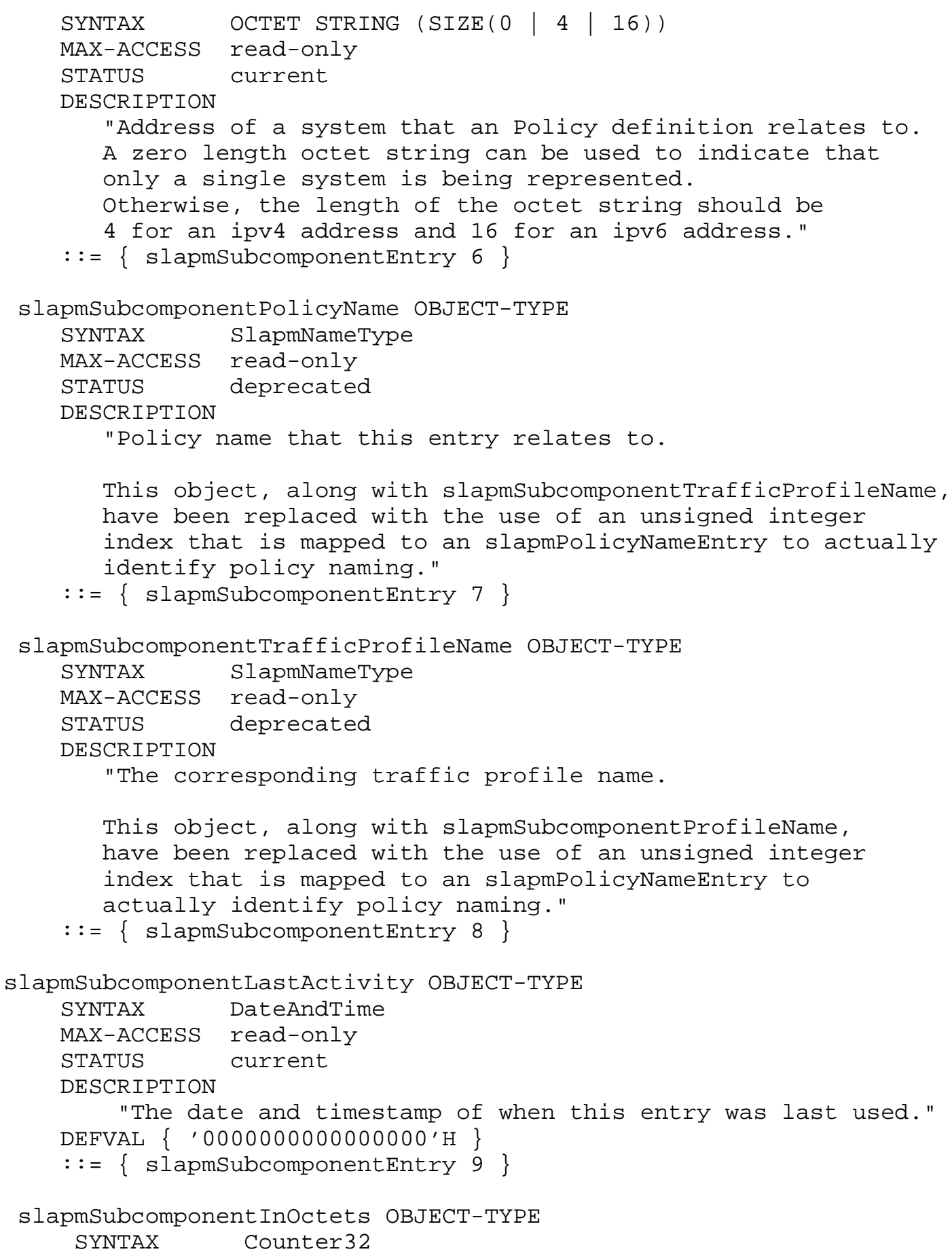




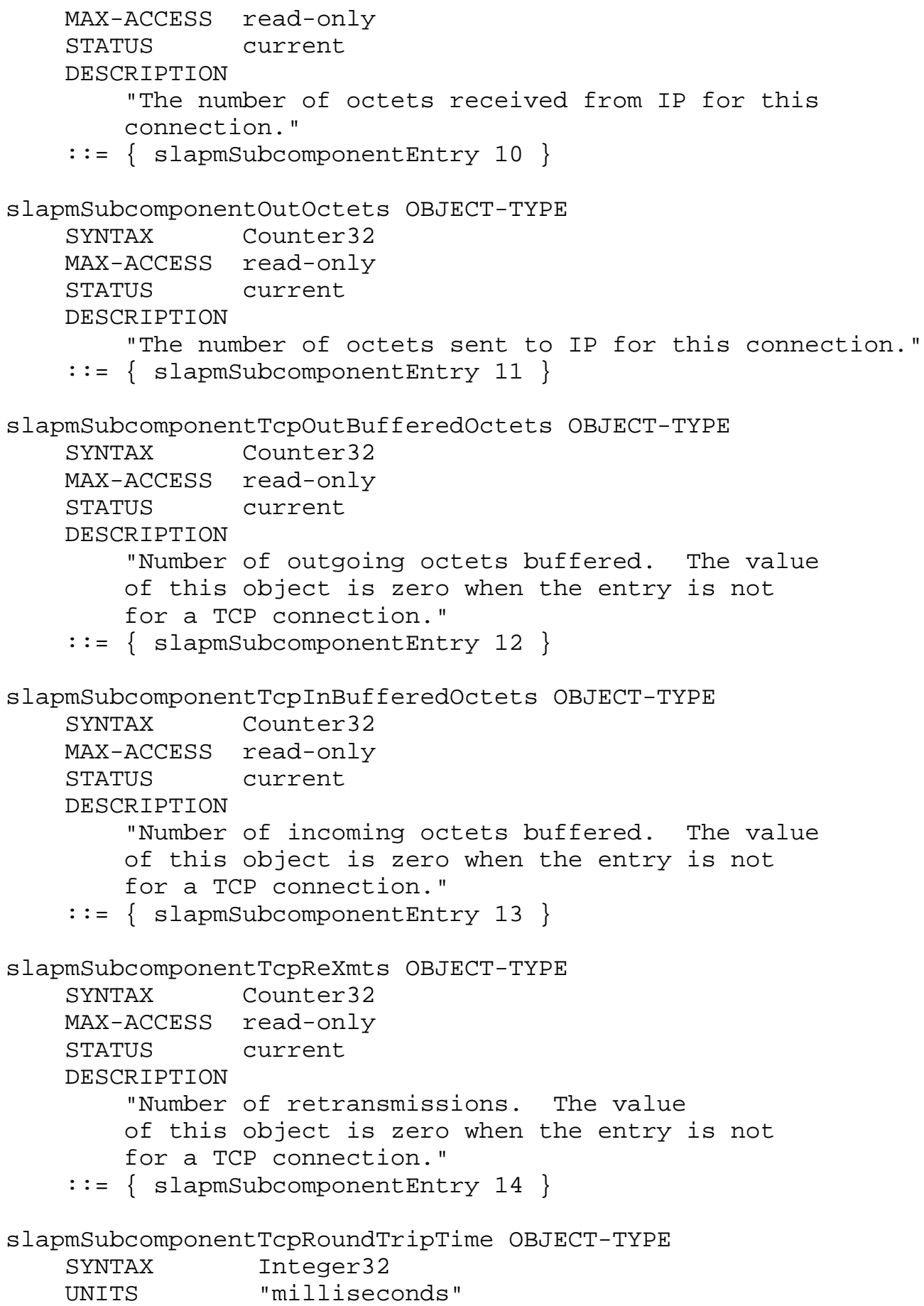




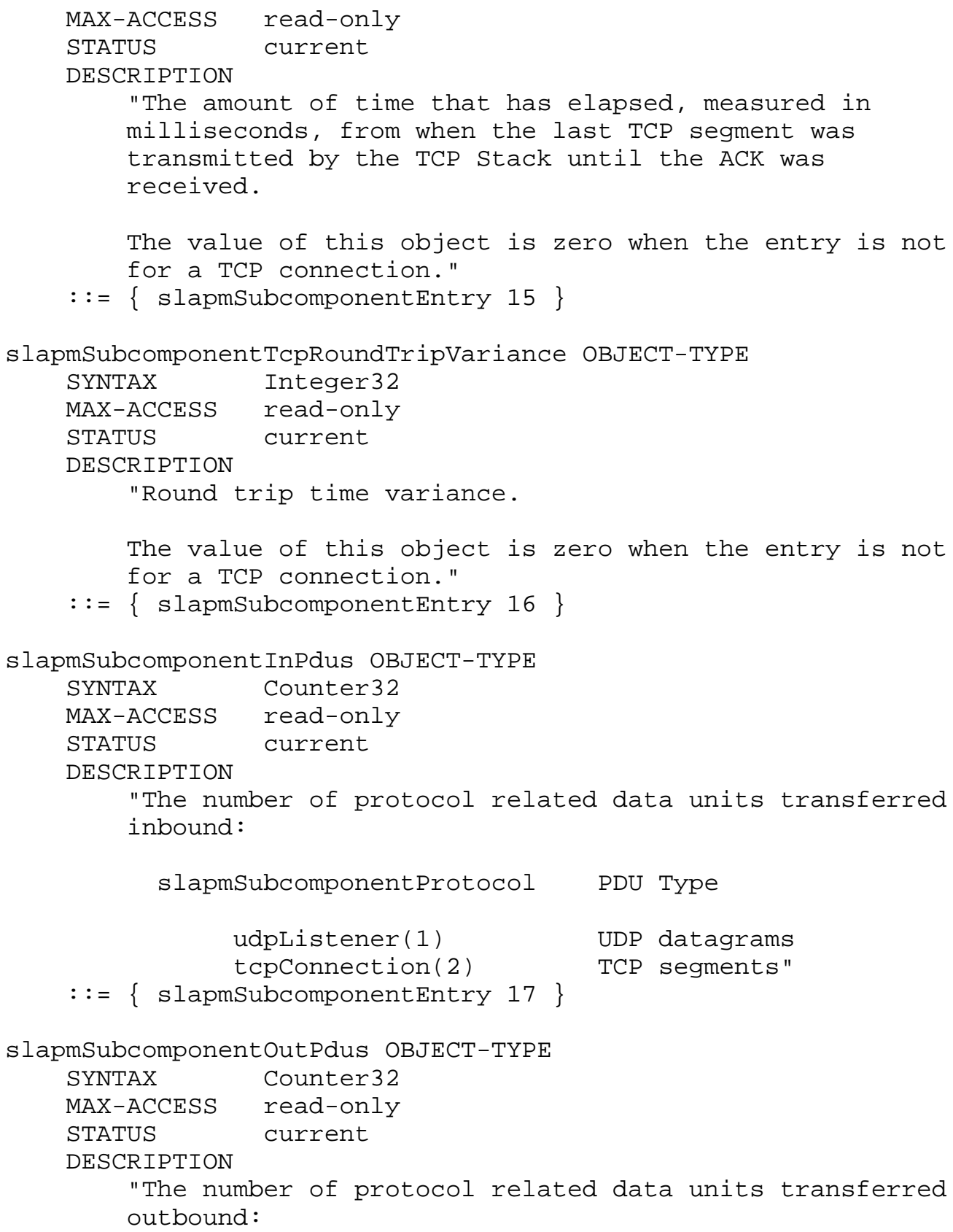




$$
::=\left\{\begin{array}{c}
\text { tcpconnection }(2) \\
\text { slapmSubcomponentEntry } 18\}
\end{array}\right. \text { TCP segments" }
$$

slapmSubcomponentApplName OBJECT-TYPE

SYNTAX SnmpAdminString (SIZE(0..32))

MAX-ACCESS read-only

STATUS current

DESCRIPTION

"The application name associated with this entry if known, otherwise a zero-length octet string is returned as the value of this object."

$::=\{$ slapmSubcomponentEntry 19$\}$

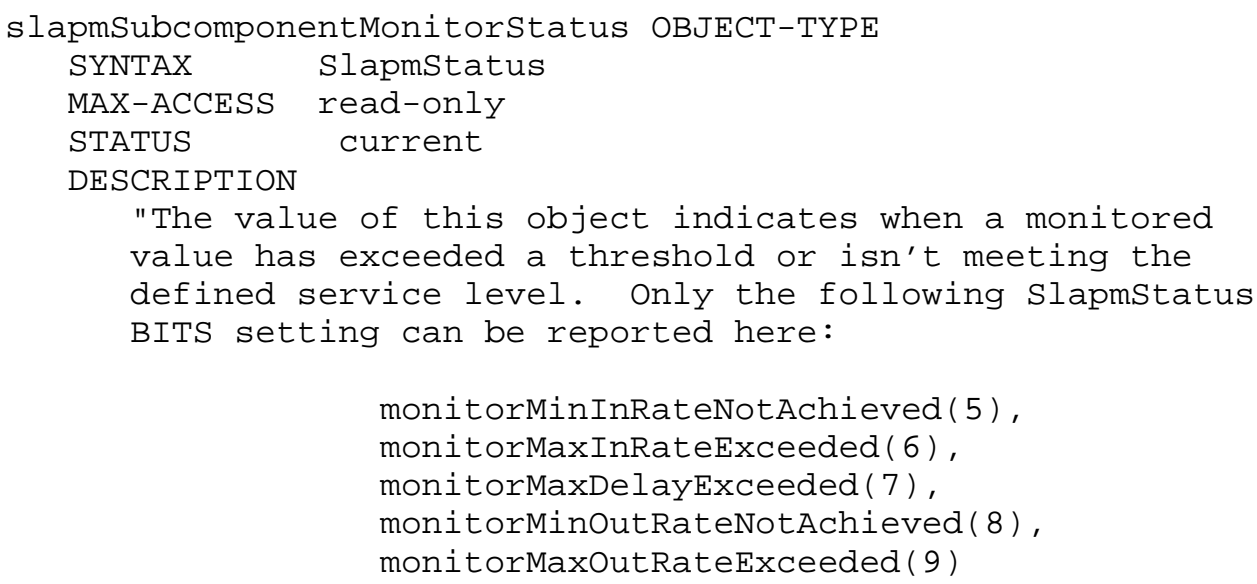

This object only has meaning when an corresponding slapmPolicyMonitorEntry exists with the slapmPolicyMonitorControl BITS setting monitorSubcomponents (5) enabled." $::=\{$ slapmSubcomponentEntry 20$\}$

slapmSubcomponentMonitorIntTime OBJECT-TYPE

$\begin{array}{ll}\text { SYNTAX } & \text { DateAndTime } \\ \text { MAX-ACCESS } & \text { read-only } \\ \text { STATUS } & \text { current }\end{array}$

DESCRIPTION

"The timestamp for when the last interval ended.

This object only has meaning when an corresponding

slapmPRMonEntry (or old slapmPolicyMonitorEntry)

exists with the slapmPRMoncontrol (or

slapmPolicyMonitorControl) BITS setting

monitorSubcomponents(5) enabled. All of the

octets returned when monitoring is not in effect 


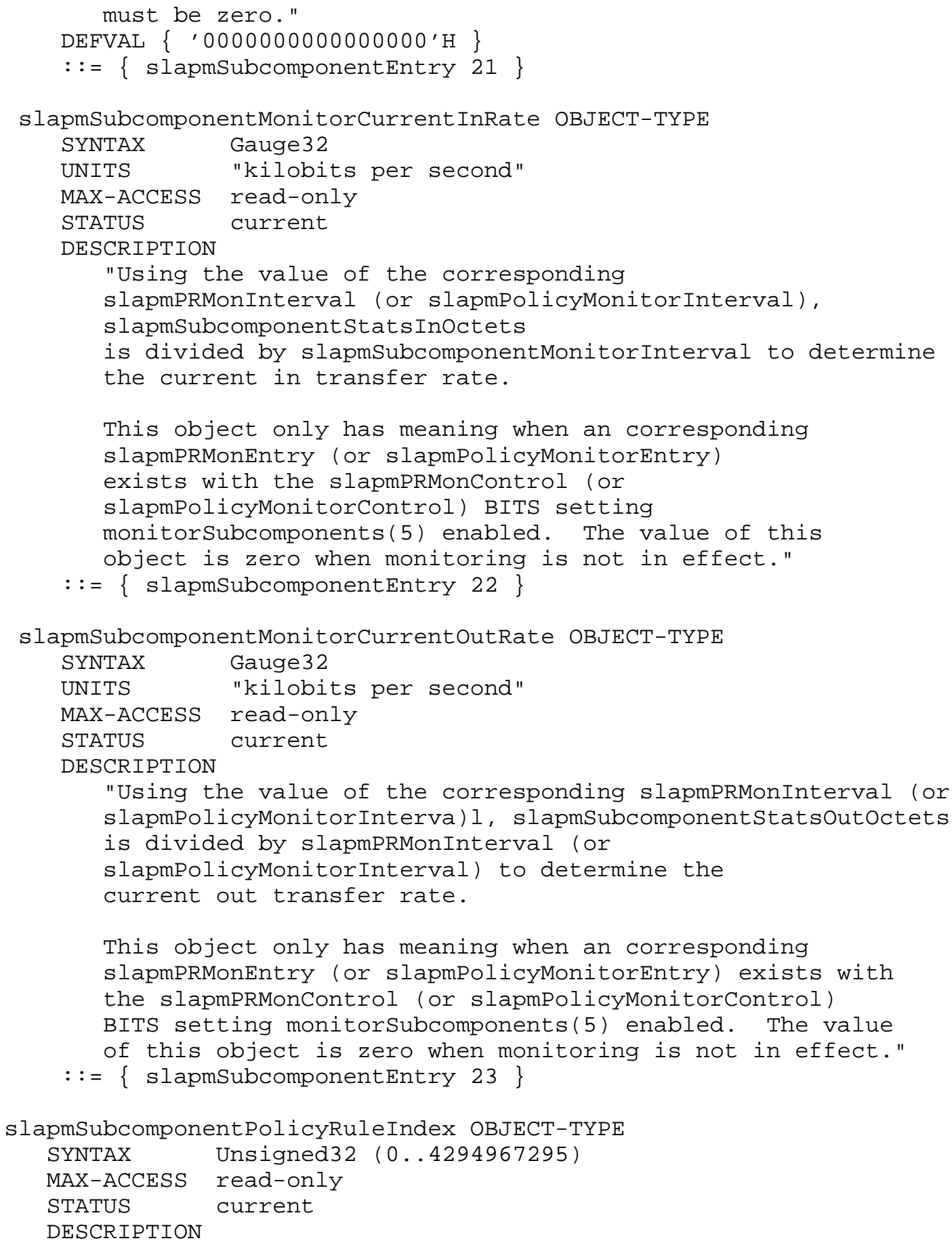




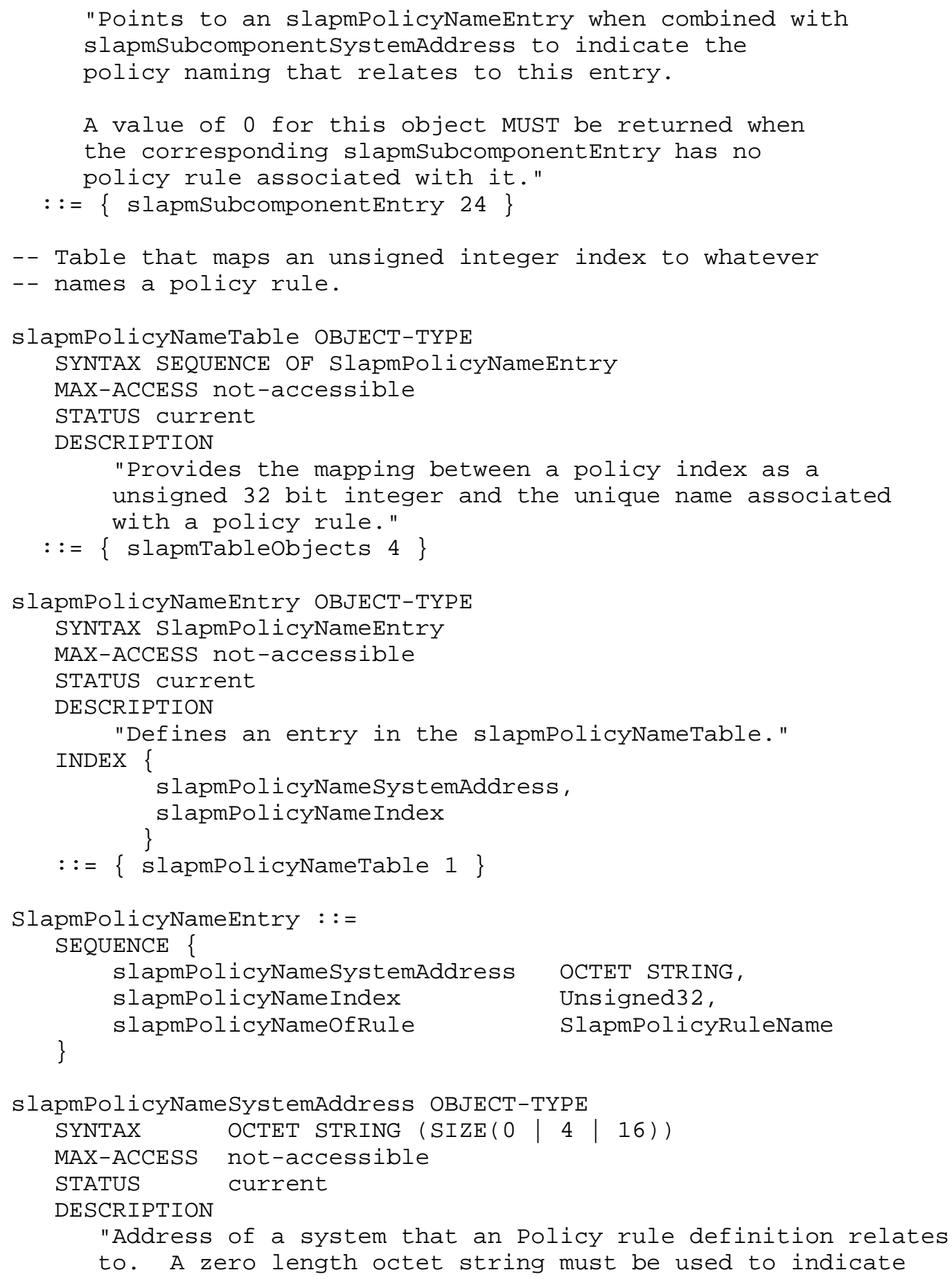




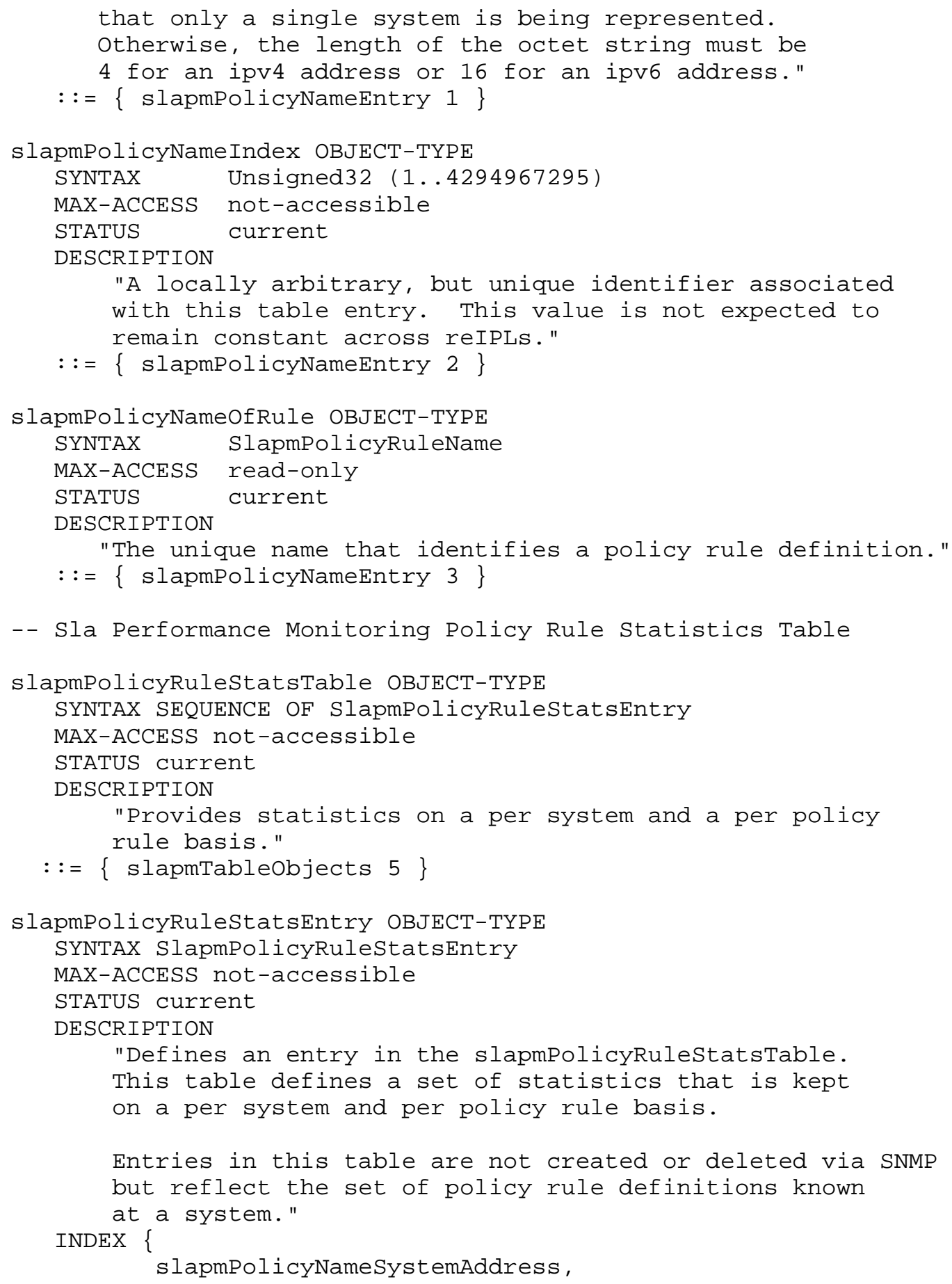




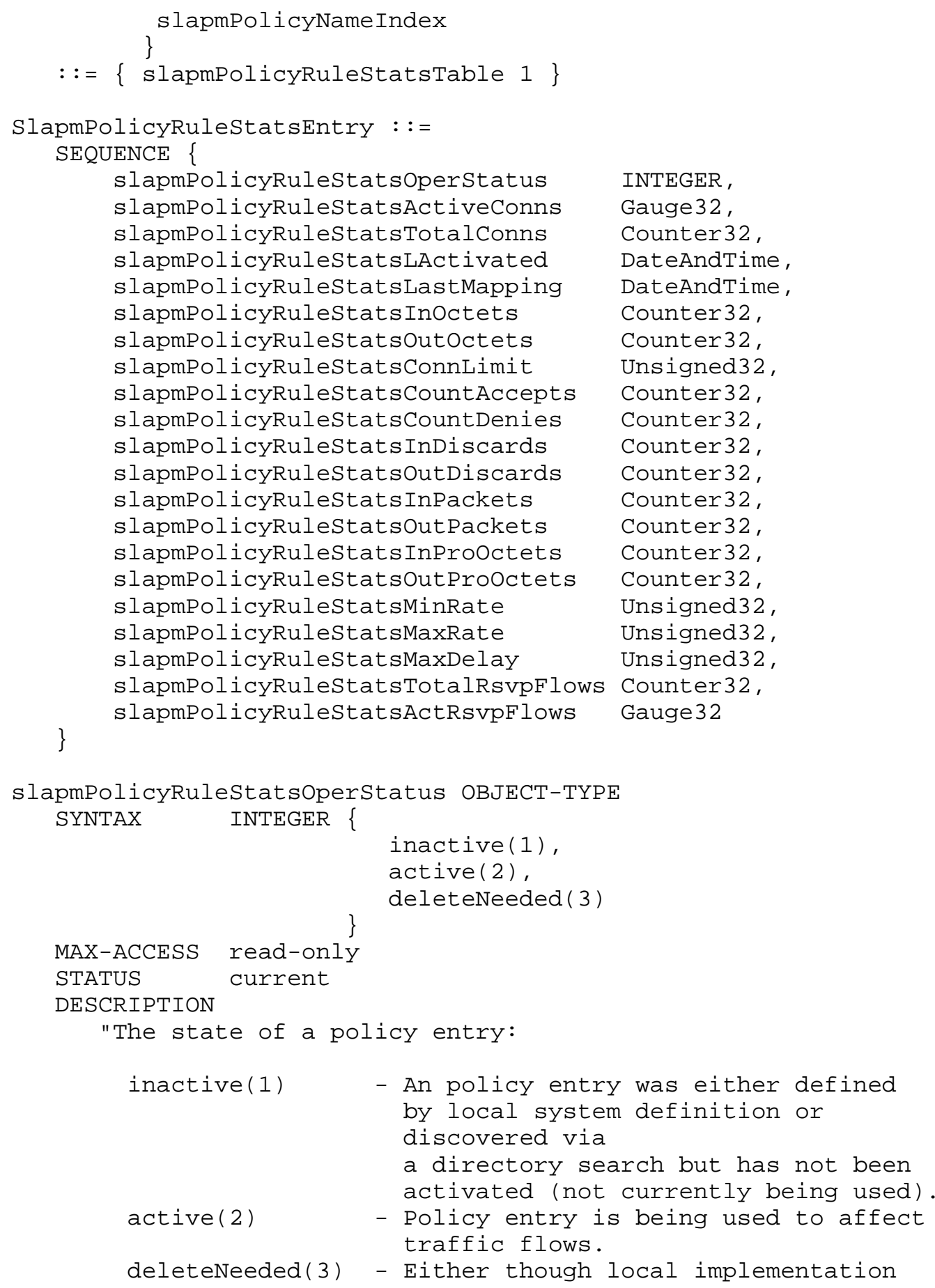




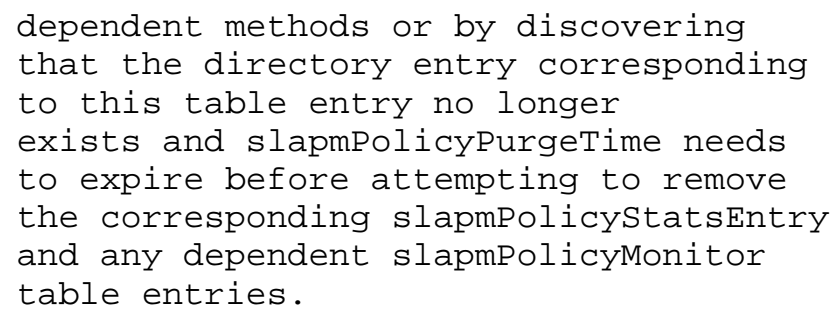




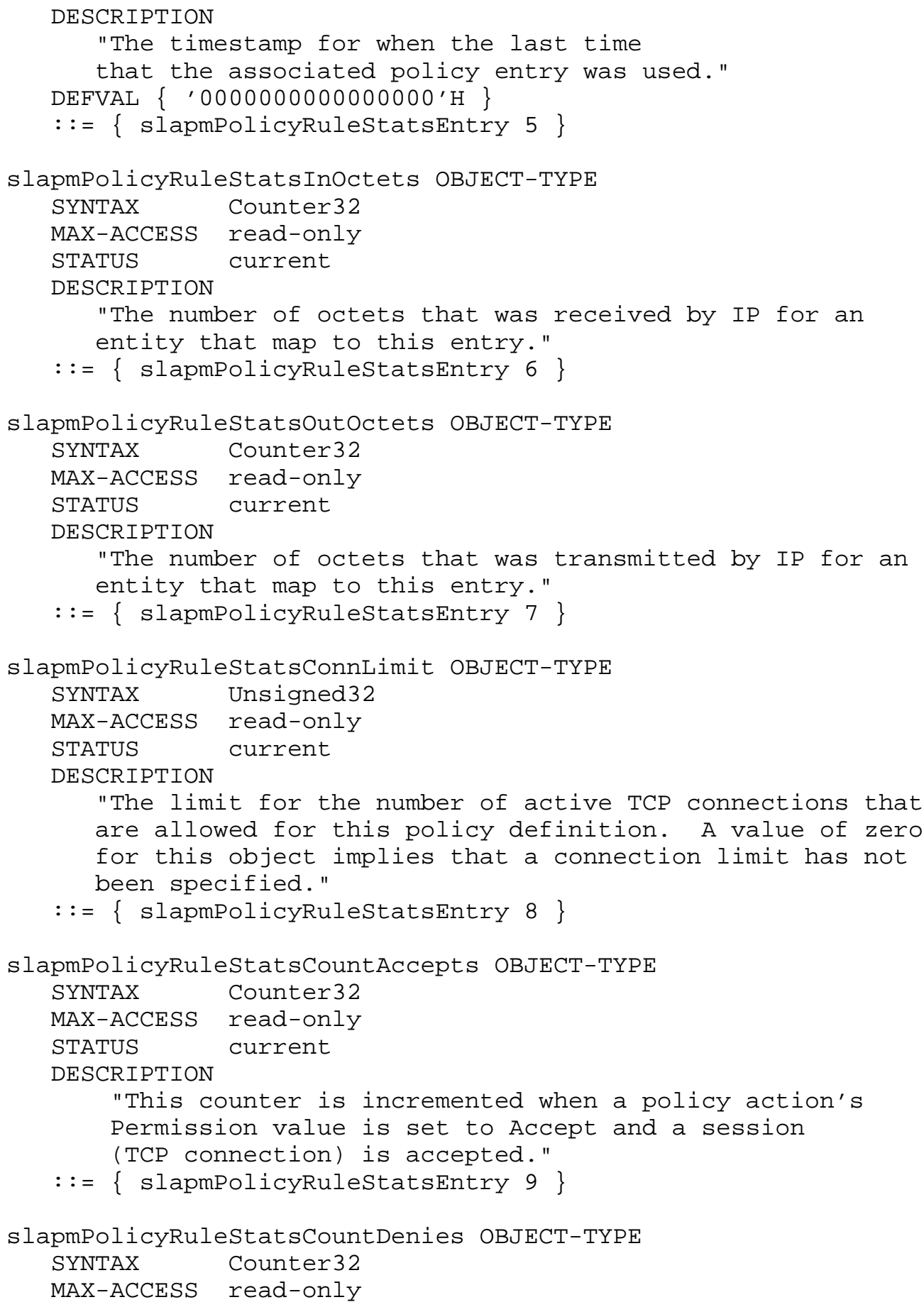




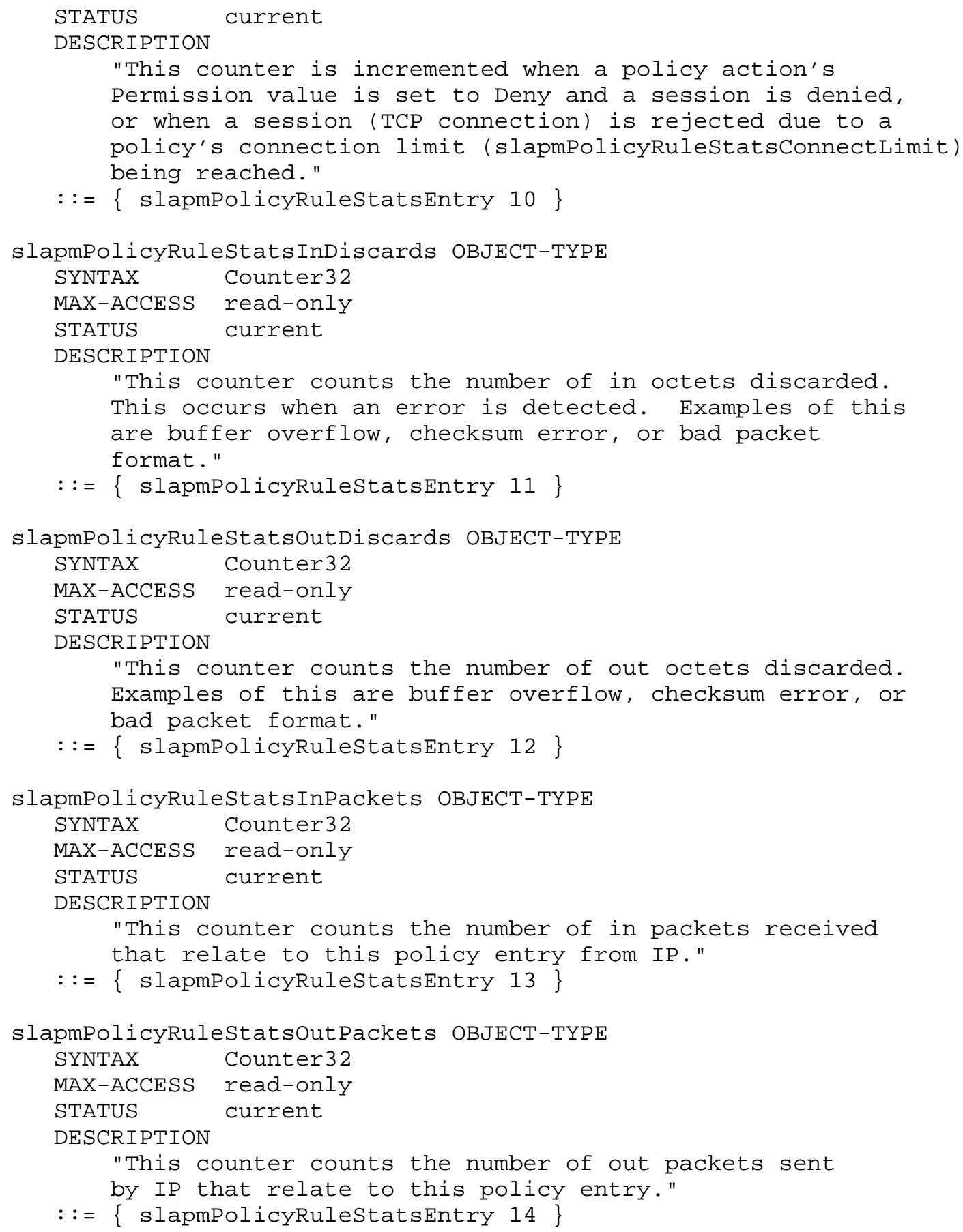




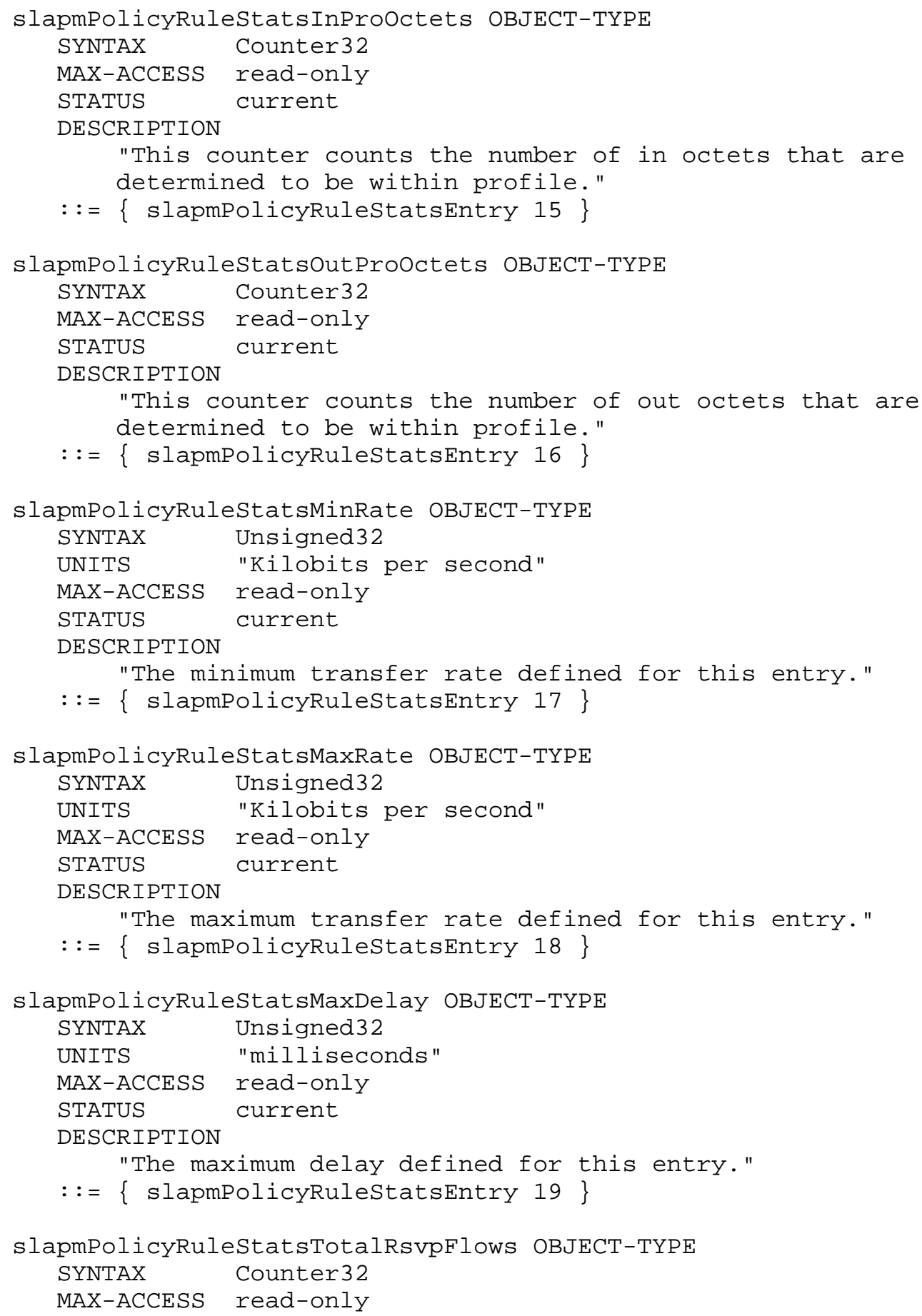




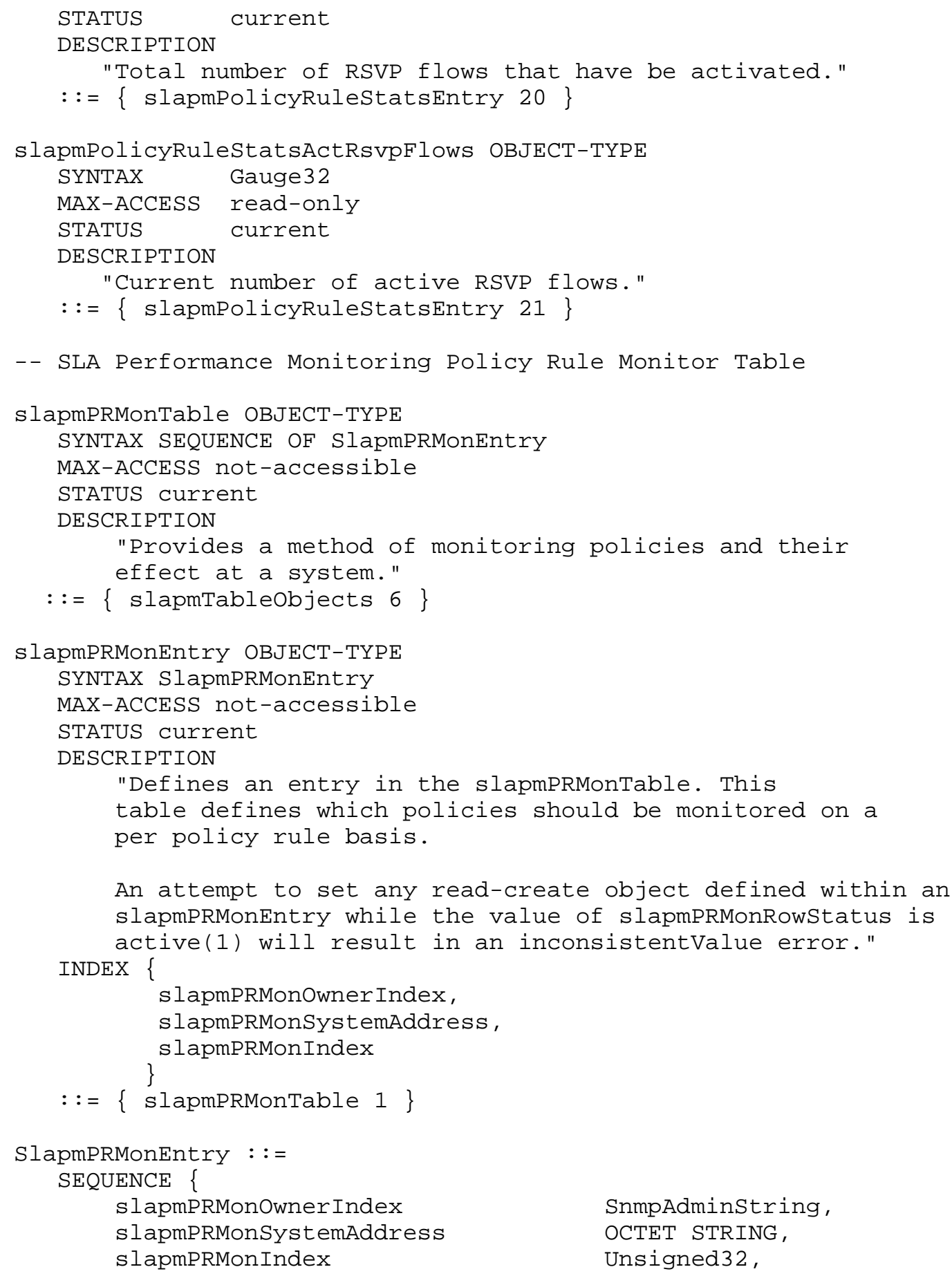




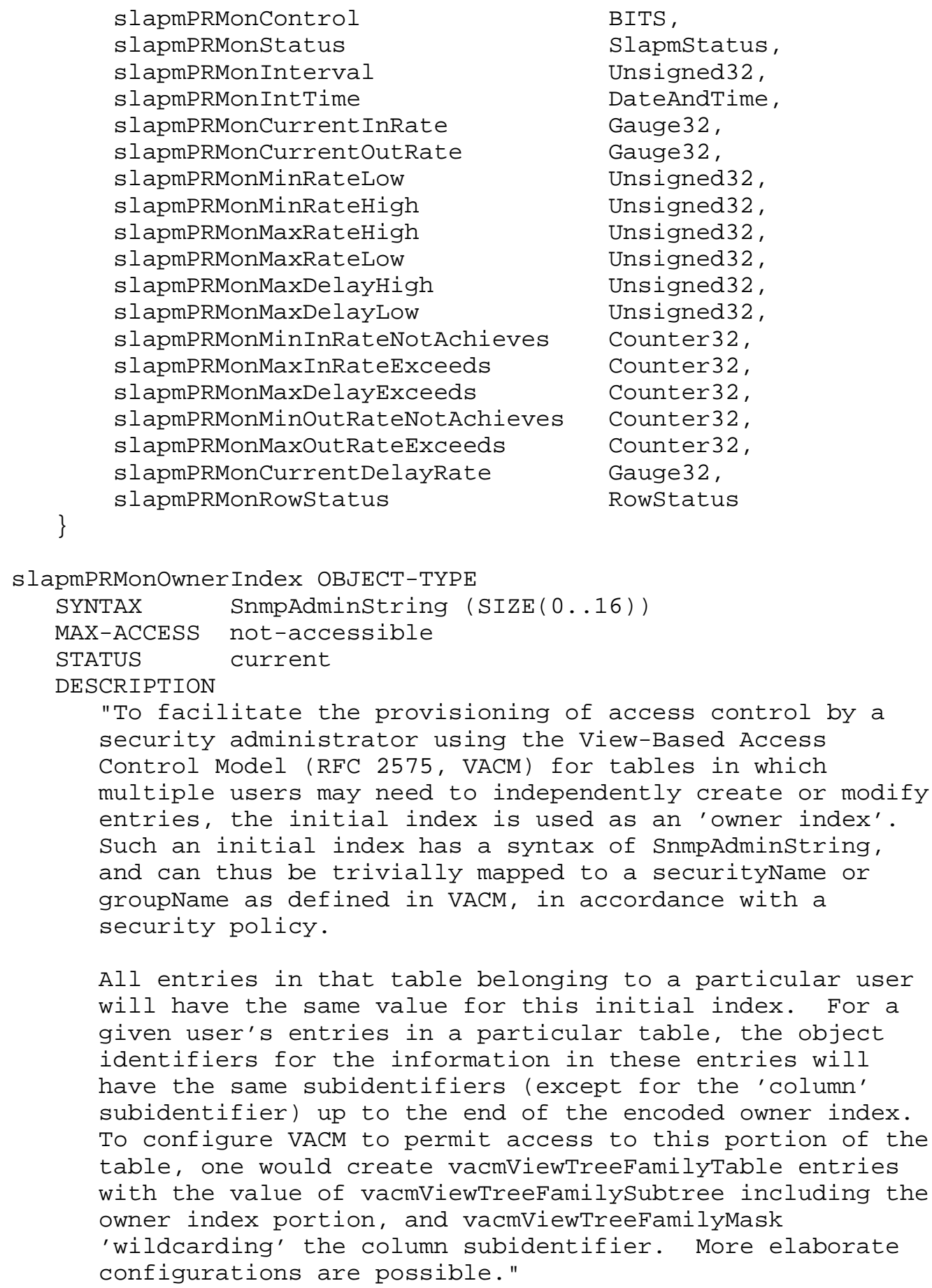

All entries in that table belonging to a particular user will have the same value for this initial index. For a given user's entries in a particular table, the object identifiers for the information in these entries will have the same subidentifiers (except for the 'column' subidentifier) up to the end of the encoded owner index. To configure VACM to permit access to this portion of the table, one would create vacmViewTreeFamilyTable entries with the value of vacmViewTreeFamilysubtree including the owner index portion, and vacmViewTreeFamilyMask

'wildcarding' the column subidentifier. More elaborate configurations are possible." 
$::=\{$ slapmPRMonEntry 1$\}$

slapmPRMonSystemAddress OBJECT-TYPE

$\left.\begin{array}{ll|l|l}\text { SYNTAX } & \text { OCTET STRING (SIZE (0 } & 4 & 16)\end{array}\right)$

STATUS current

DESCRIPTION

"Address of a system that an Policy definition relates to.

A zero length octet string can be used to indicate that

only a single system is being represented.

Otherwise, the length of the octet string should be

4 for an ipv4 address and 16 for an ipv6 address."

$::=\{$ slapmPRMonEntry 2$\}$

slapmPRMonIndex OBJECT-TYPE

SYNTAX Unsigned32

MAX-ACCESS not-accessible

STATUS current

DESCRIPTION

"An slapmPolicyNameTable index, slapmPolicyNameIndex,

that points to the unique name associated with a

policy rule definition."

$::=\{$ slapmPRMonEntry 3$\}$

slapmPRMonControl OBJECT-TYPE

SYNTAX BITS \{

monitorMinRate (0),

monitorMaxRate (1),

monitorMaxDelay (2),

enableAggregateTraps (3),

enableSubcomponent Traps (4),

monitorSubcomponents (5) \}

MAX-ACCESS read-create

STATUS current

DESCRIPTION

"The value of this object determines the type and level

of monitoring that is applied to a policy rule. The

value of this object can't be changed once the table

entry that it is a part of is activated via a

slapmPRMonRowstatus transition to active state.

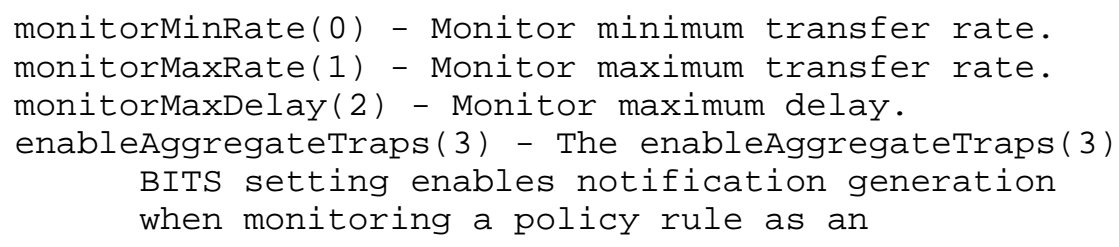




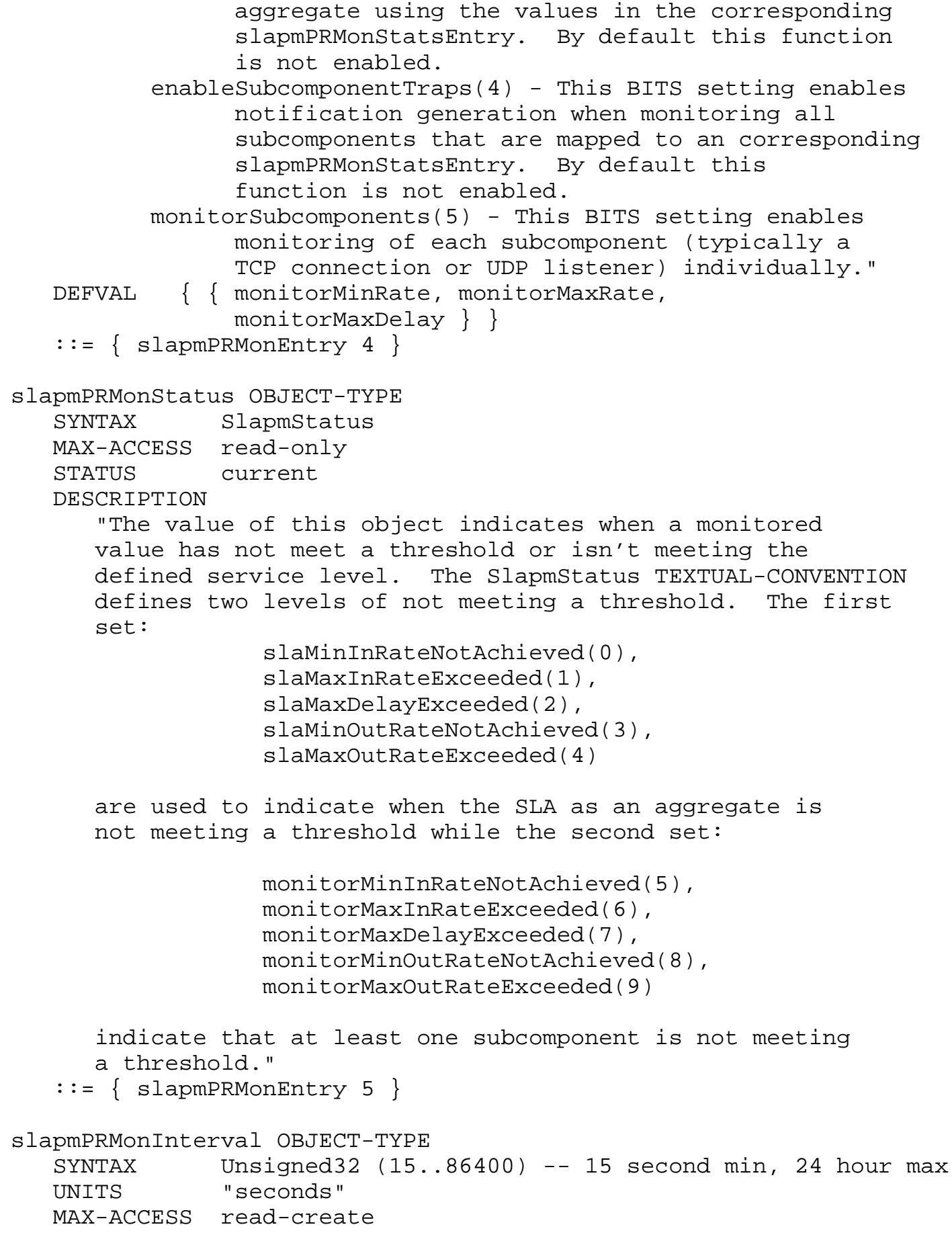




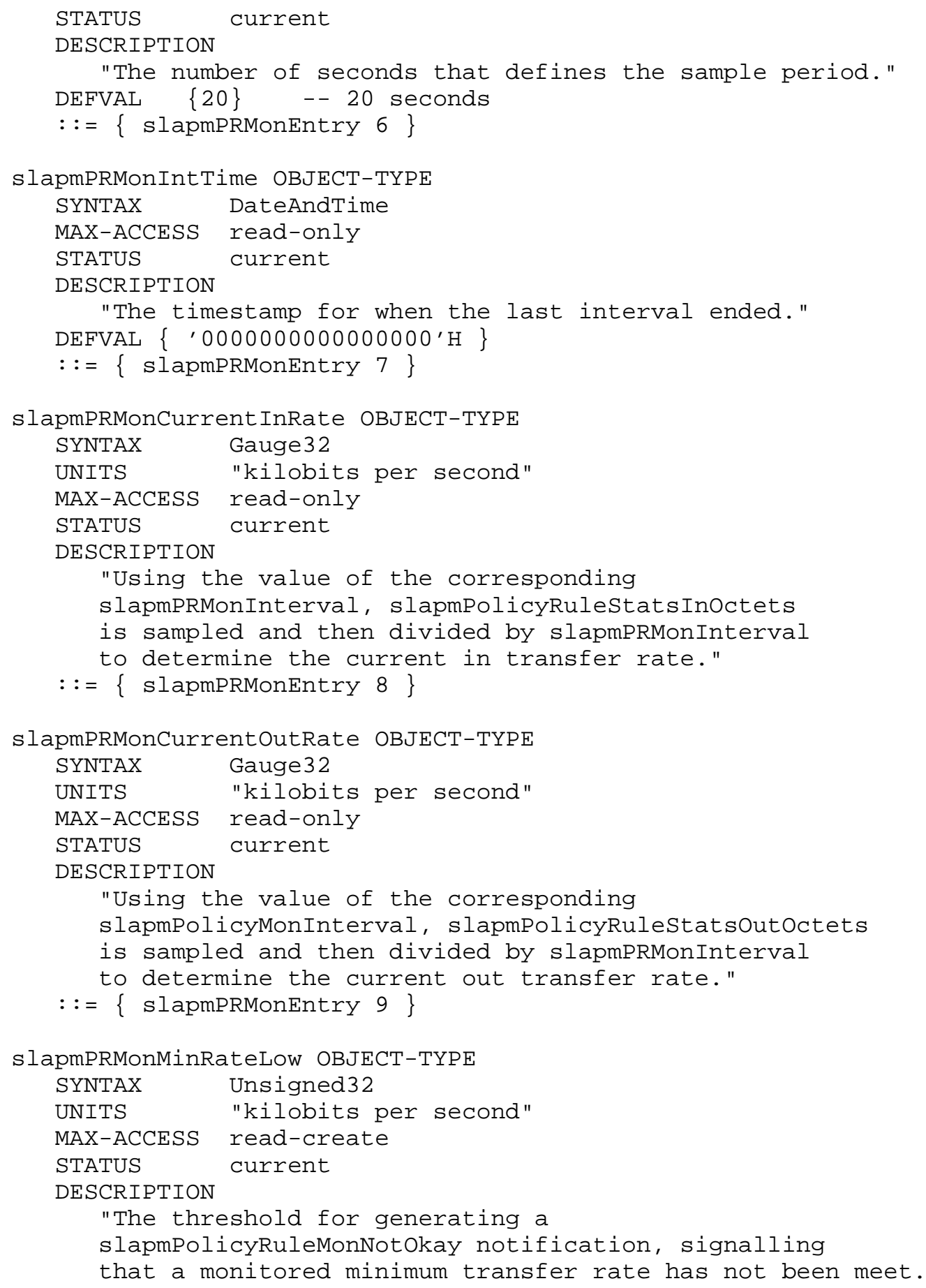


A slapmPolicyRuleMonNotokay notification is not generated again for an slapmPRMonEntry until the minimum transfer rate exceeds slapmPRMonMinRateHigh (a slapmPolicyRuleMonokay notification is then transmitted) and then fails below slapmPRMonMinRatelow. This behavior reduces the slapmPolicyRuleMonNotokay notifications that are transmitted.

A value of zero for this object is returned when the slapmPRMonControl monitorMinRate(0) is not enabled. When enabled the default value for this object is the min rate value specified in the associated action definition minus 10\%. If the action definition doesn't have a min rate defined then there is no default for this object and a value MUST be specified prior to activating this entry when monitorMinRate(0) is selected.

Note: The corresponding slapmPRMonControl

BITS setting, enableAggregateTraps(3), MUST be selected in order for any notification relating to this entry to potentially be generated."

$::=\{$ slapmPRMonEntry 10$\}$

slapmPRMonMinRateHigh OBJECT-TYPE

SYNTAX Unsigned32

UNITS "kilobits per second"

MAX-ACCESS read-create

STATUS current

DESCRIPTION

"The threshold for generating a slapmPolicyRuleMonokay notification, signalling that a monitored minimum transfer rate has increased to an acceptable level.

A value of zero for this object is returned when the slapmPRMonControl monitorMinRate(0) is not enabled. When enabled the default value for this object is the min rate value specified in the associated action definition plus $10 \%$. If the action definition doesn't have a min rate defined then there is no default for this object and a value MUST be specified prior to activating this entry when monitorMinRate(0) is selected.

Note: The corresponding slapmPRMonControl BITS setting, enableAggregateTraps(3), MUST be selected in order for any notification relating to this entry to 


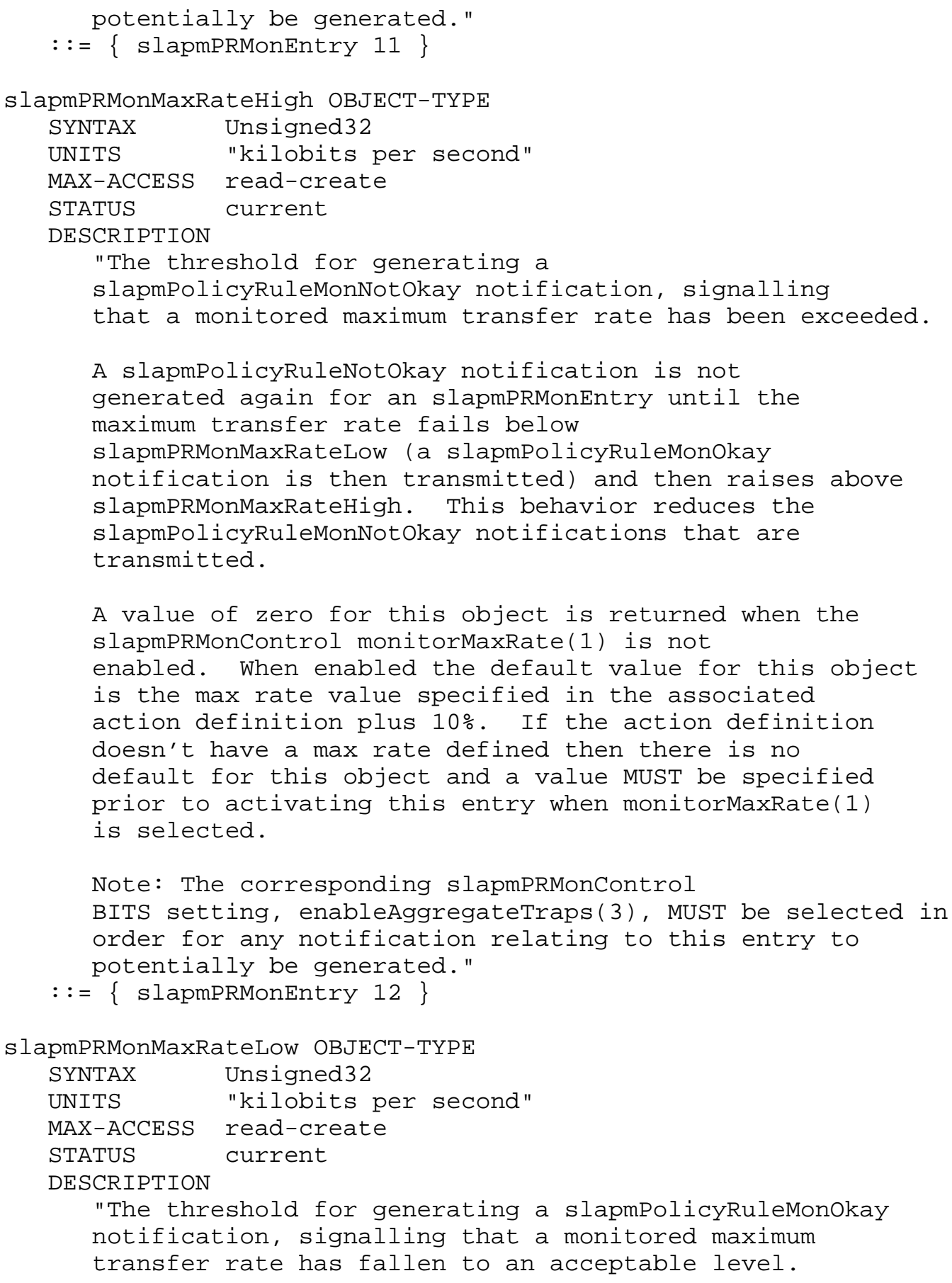


A value of zero for this object is returned when the slapmPRMonControl monitorMaxRate(1) is not enabled. When enabled the default value for this object is the max rate value specified in the associated action definition minus $10 \%$. If the action definition doesn't have a max rate defined then there is no default for this object and a value MUST be specified prior to activating this entry when monitorMaxRate(1) is selected.

Note: The corresponding slapmPRMonControl

BITS setting, enableAggregateTraps(3), MUST be selected in order for any notification relating to this entry to potentially be generated." $::=\{$ slapmPRMonEntry 13$\}$

slapmPRMonMaxDelayHigh OBJECT-TYPE

SYNTAX Unsigned32

UNITS "milliseconds"

MAX-ACCESS read-create

STATUS current

DESCRIPTION

"The threshold for generating a

slapmPolicyRuleMonNotokay notification, signalling

that a monitored maximum delay rate has been exceeded.

A slapmPolicyRuleMonNotokay notification is not generated again for an slapmPRMonEntry until the maximum delay rate falls below slapmPRMonMaxDelayLow (a slapmPolicyRuleMonokay notification is then transmitted) and raises above slapmPRMonMaxDelayHigh. This behavior reduces the slapmPolicyRuleMonNotokay notifications that are transmitted.

A value of zero for this object is returned when the slapmPRMonControl monitorMaxDelay(4) is not enabled. When enabled the default value for this object is the max delay value specified in the associated action definition plus $10 \%$. If the action definition doesn't have a max delay defined then there is no default for this object and a value MUST be specified prior to activating this entry when monitorMaxDelay (4) is selected.

Note: The corresponding slapmPRMonControl BITS setting, enableAggregateTraps(3), MUST be selected in order for any notification relating to this entry to 


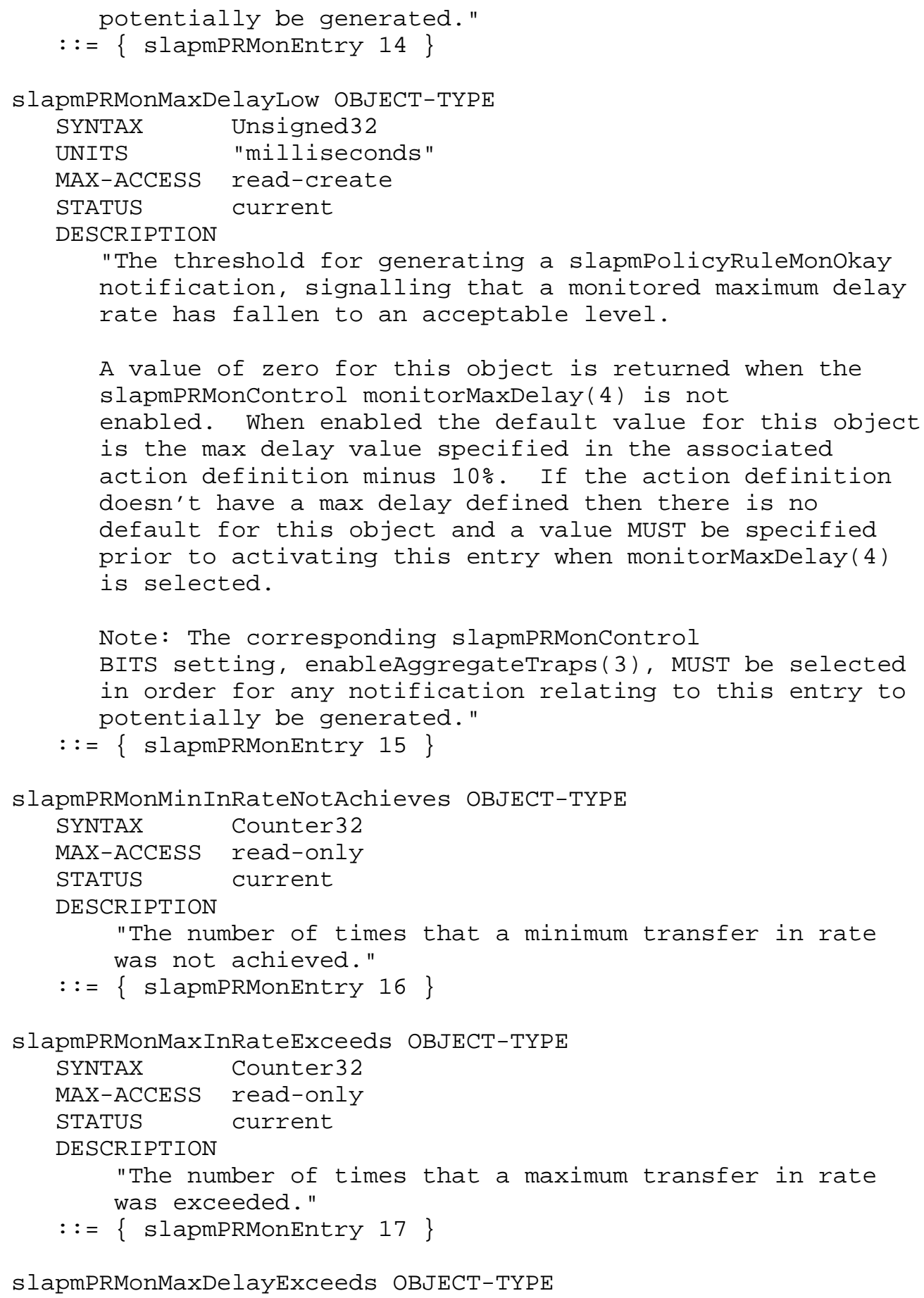




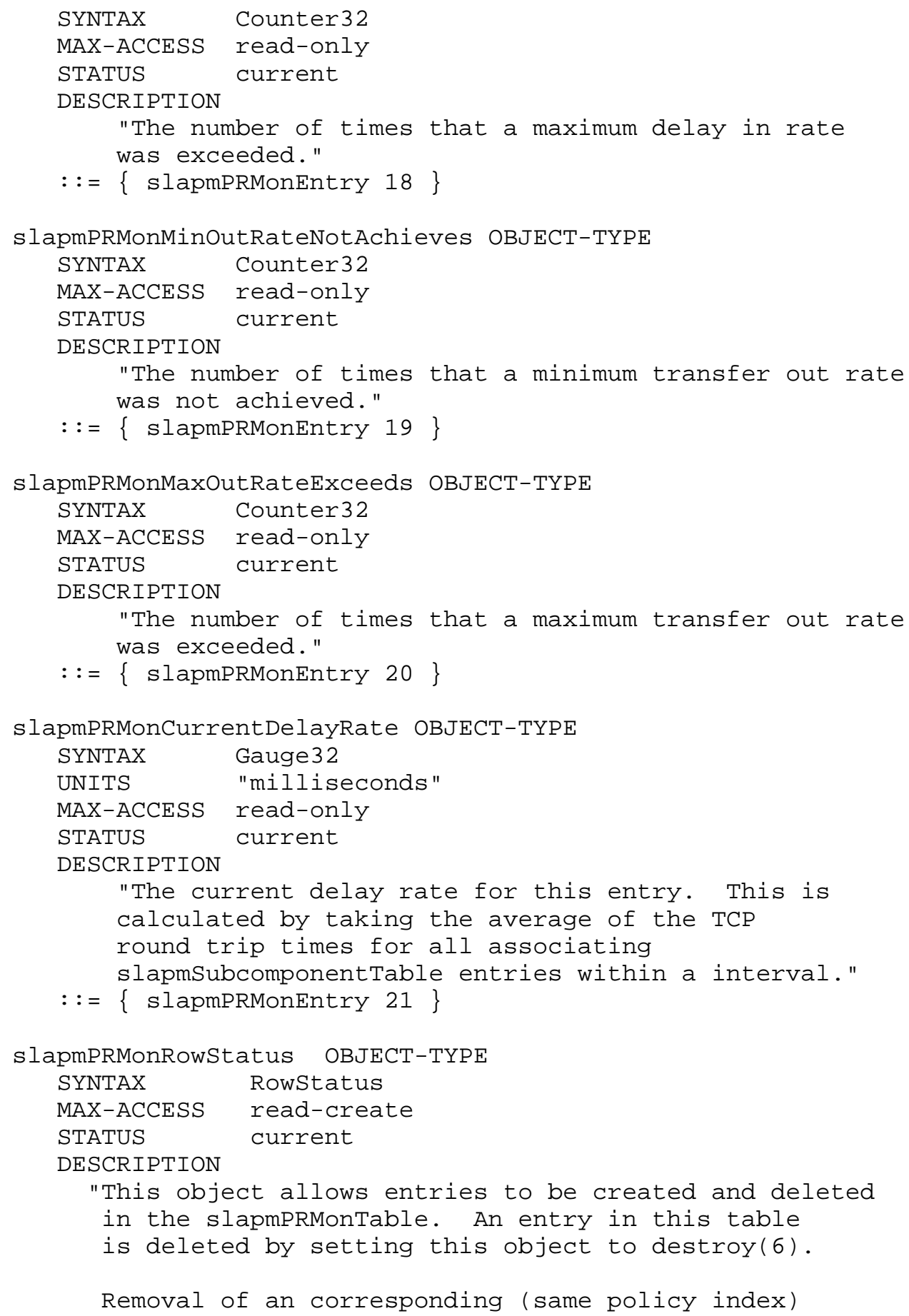




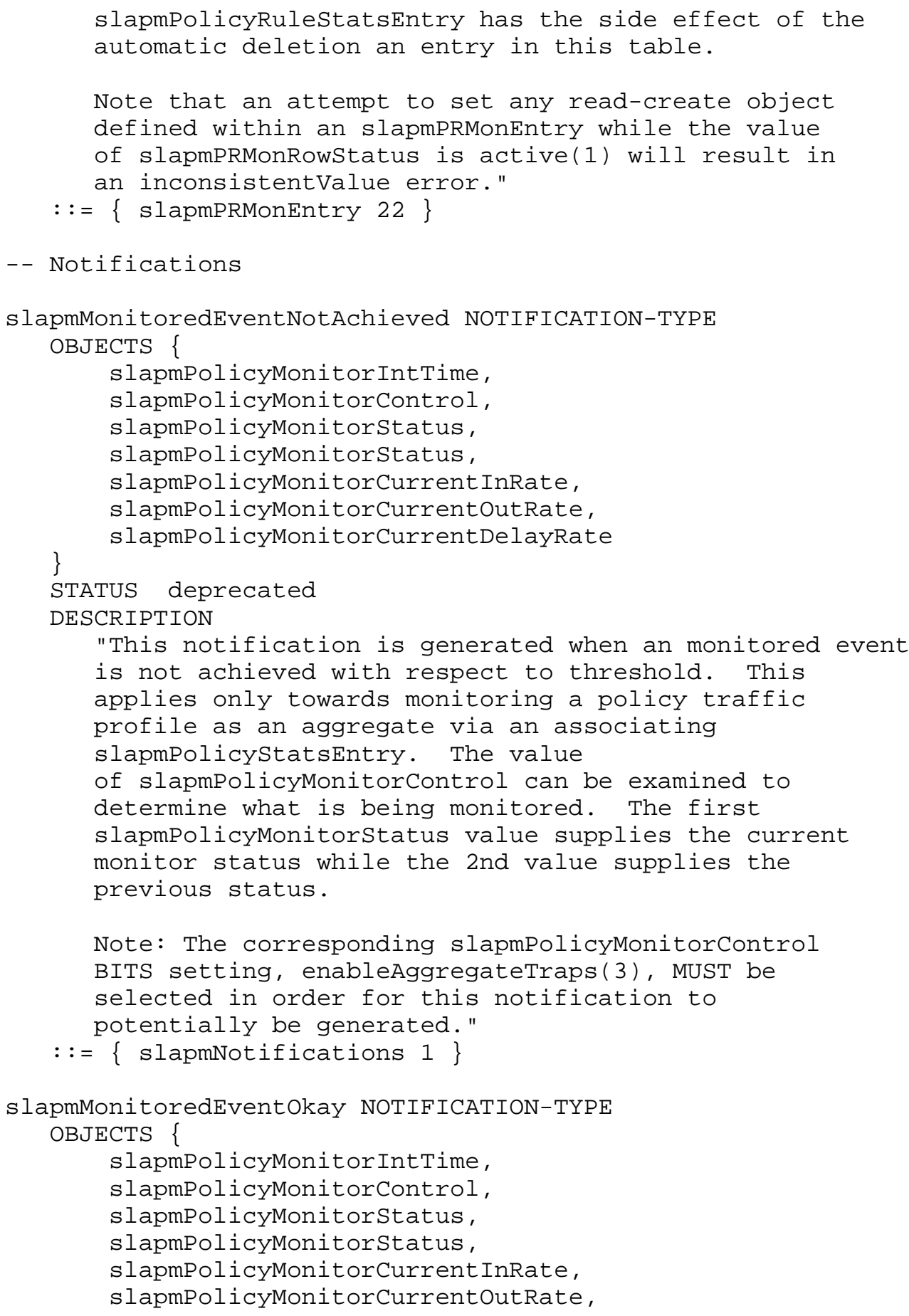




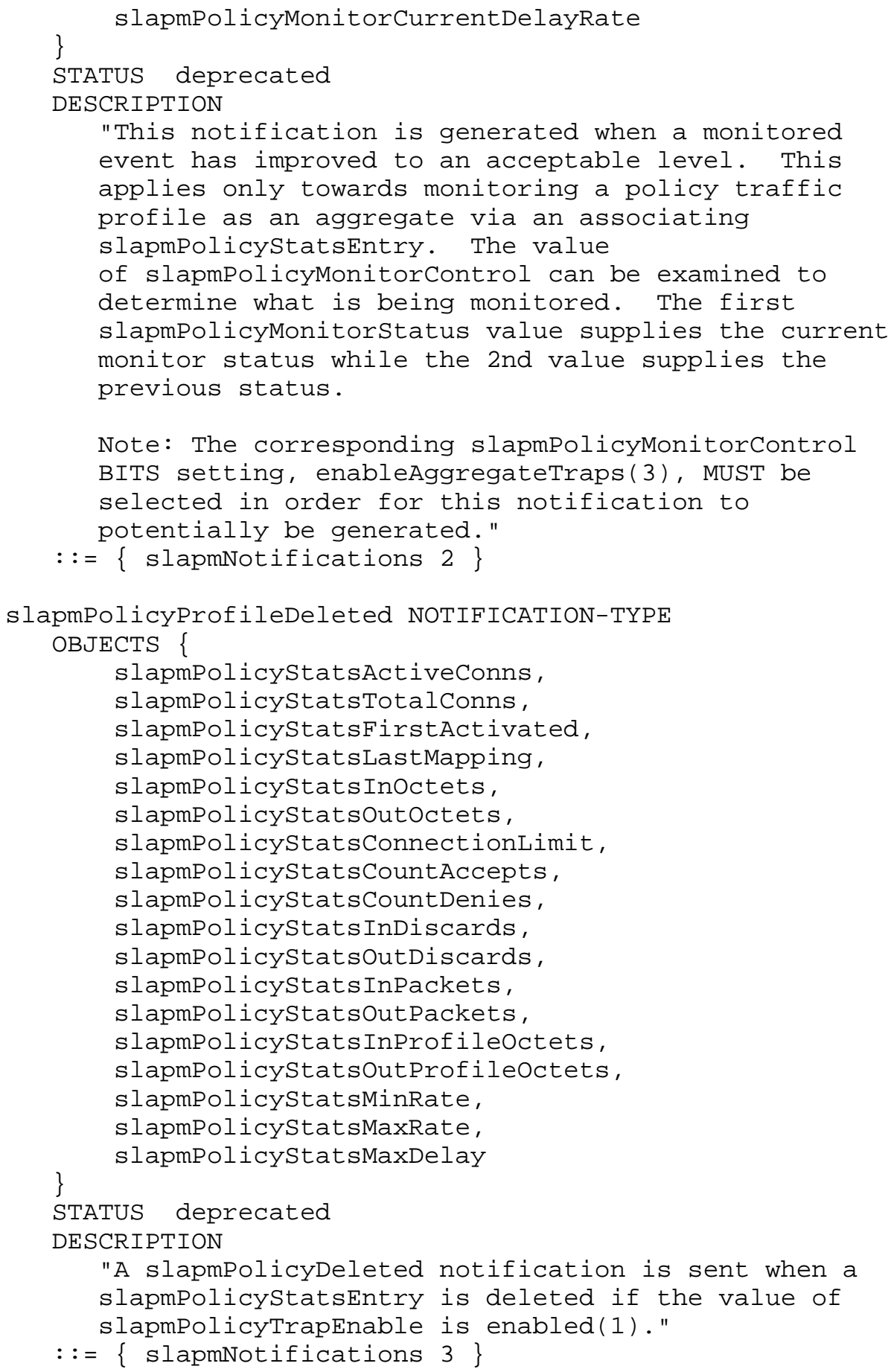

"A slapmPolicyDeleted notification is sent when a slapmPolicystatsEntry is deleted if the value of 


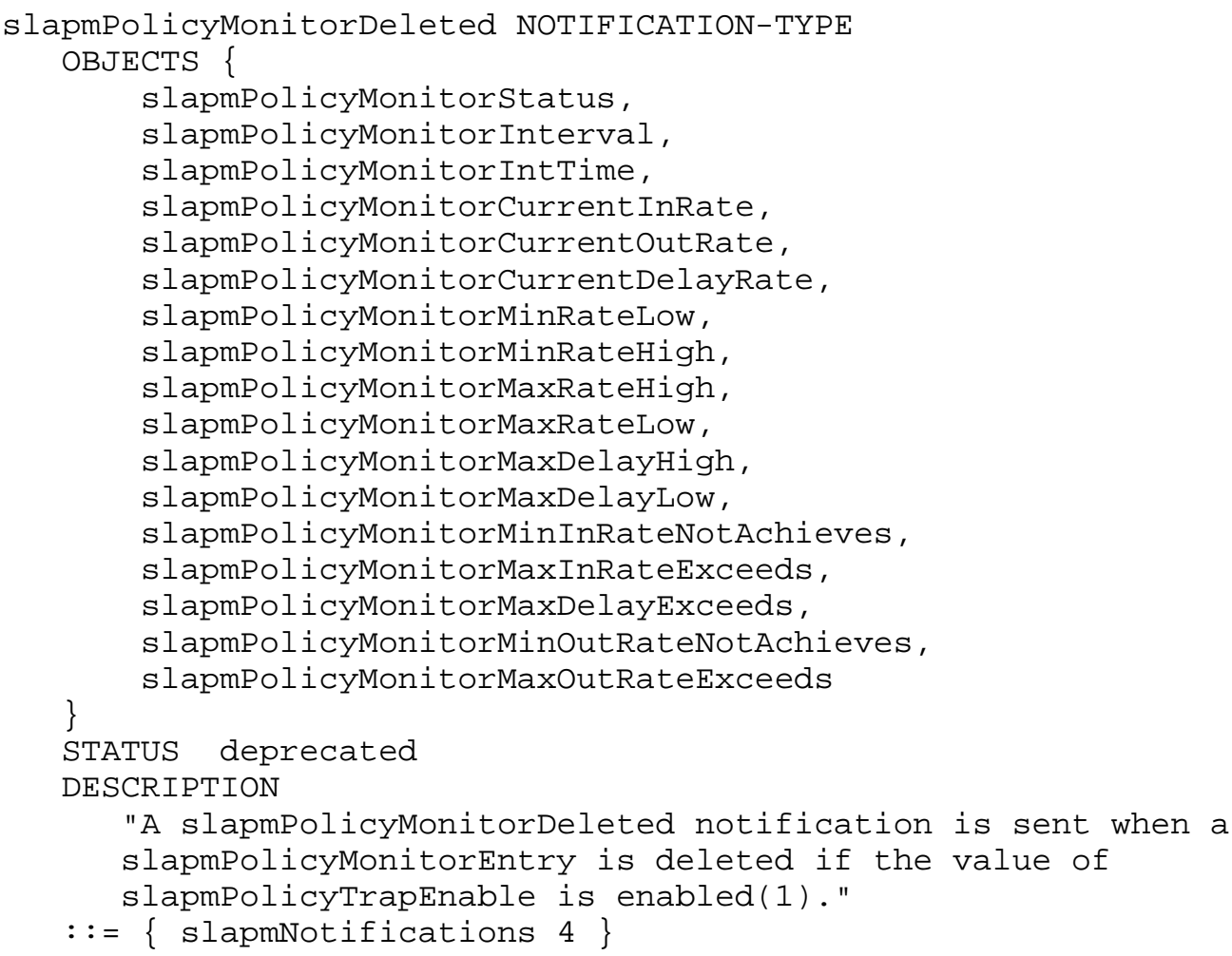

slapmSubcomponentMonitoredEventNotAchieved NOTIFICATION-TYPE OBJECTS \{

slapmSubcomponent SystemAddress,

slapmSubcomponentPolicyName,

slapmSubcomponentTrafficProfileName,

slapmSubcomponentMonitorstatus,

slapmSubcomponentMonitorStatus,

slapmSubcomponentMonitorIntTime,

slapmSubcomponentMonitorCurrentInRate, slapmSubcomponentMonitorCurrentoutRate,

"This notification is generated when a monitored value does not achieved a threshold specification. This applies only towards monitoring the individual components of a policy traffic profile. The value of the corresponding slapmPolicyMonitorControl can be examined to determine what is being monitored. The first slapmSubcomponentMonitorstatus value supplies the current 


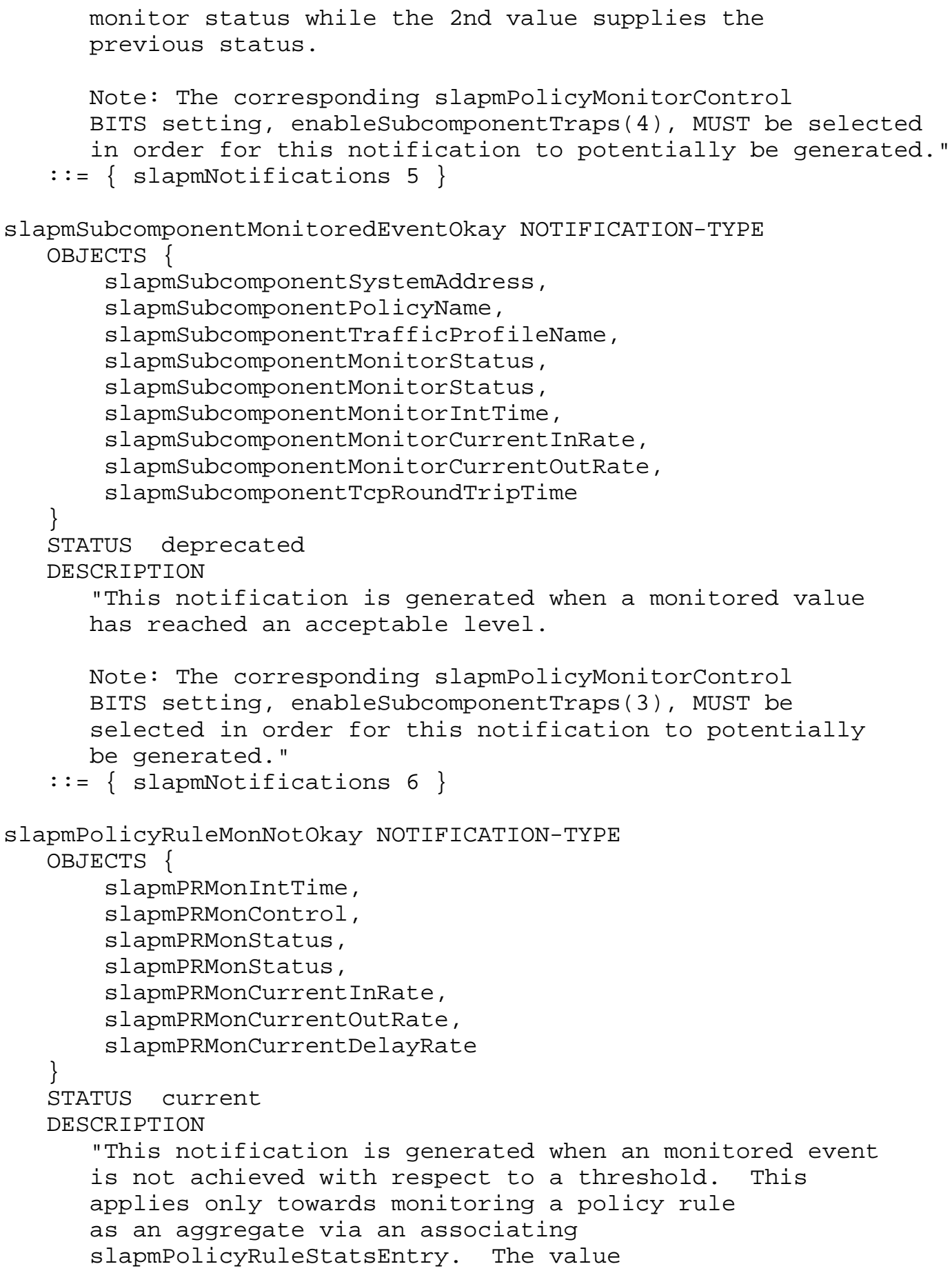




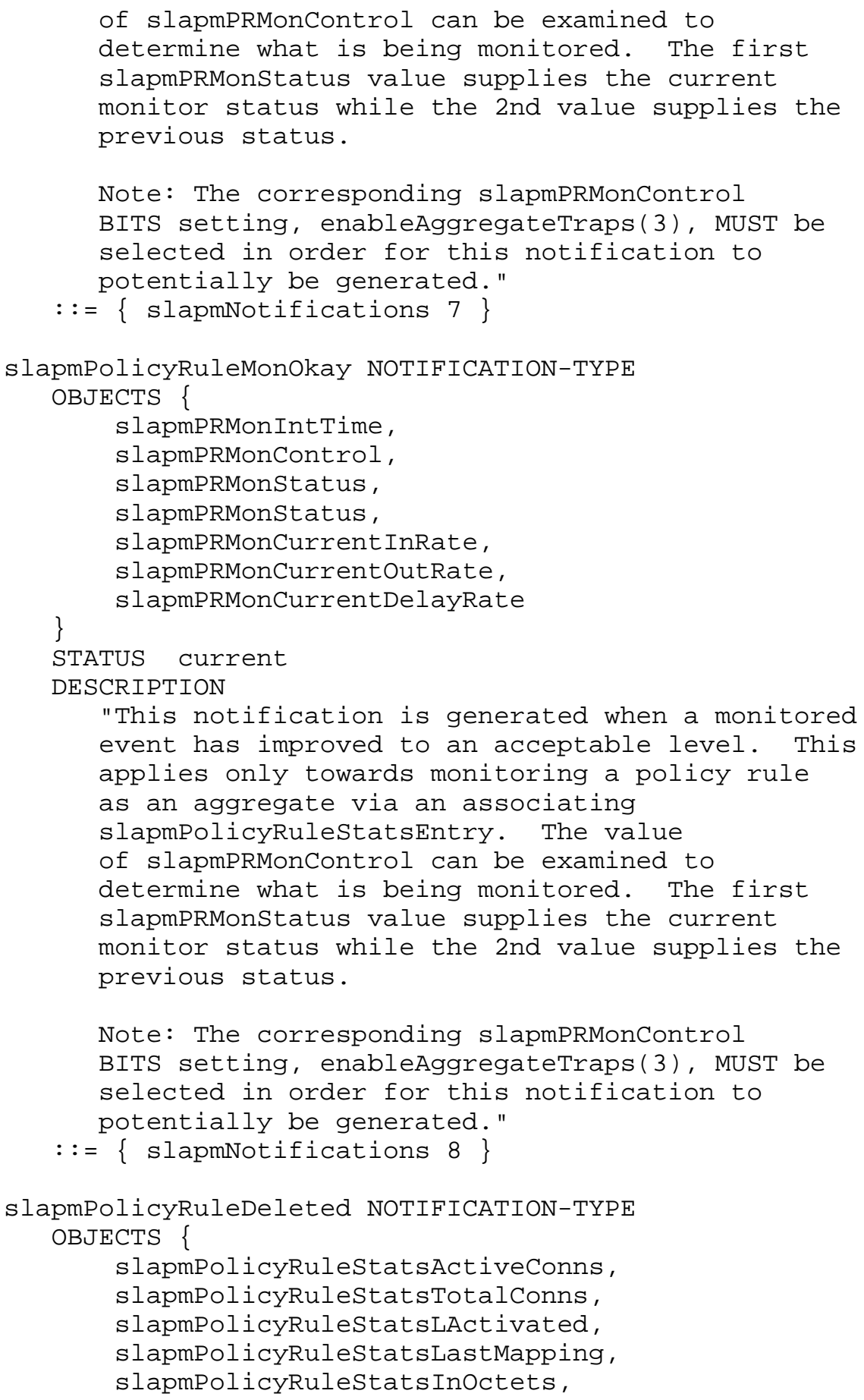




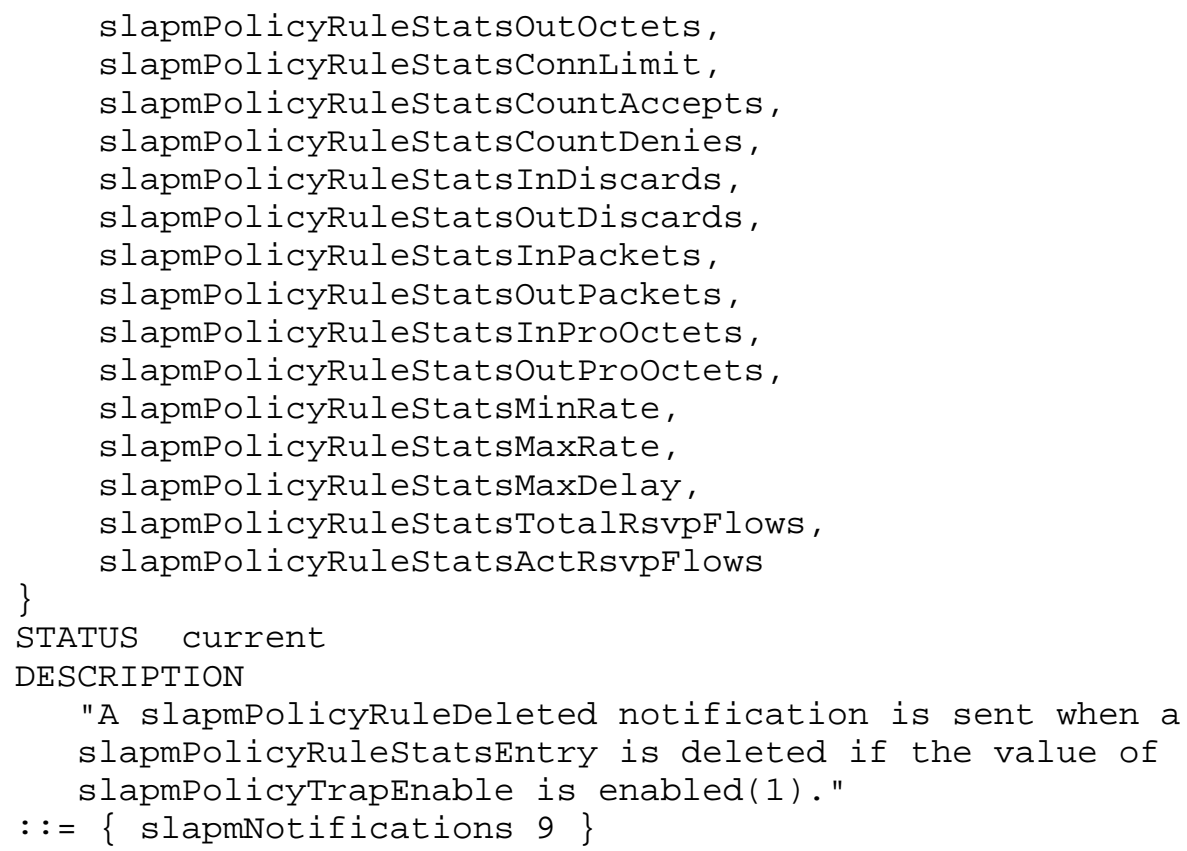

"A slapmPolicyRuleMonDeleted notification is sent when a slapmPRMonEntry is deleted if the value of 


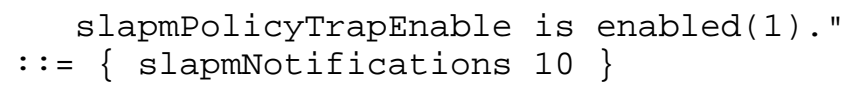

"This notification is generated when a monitored value 


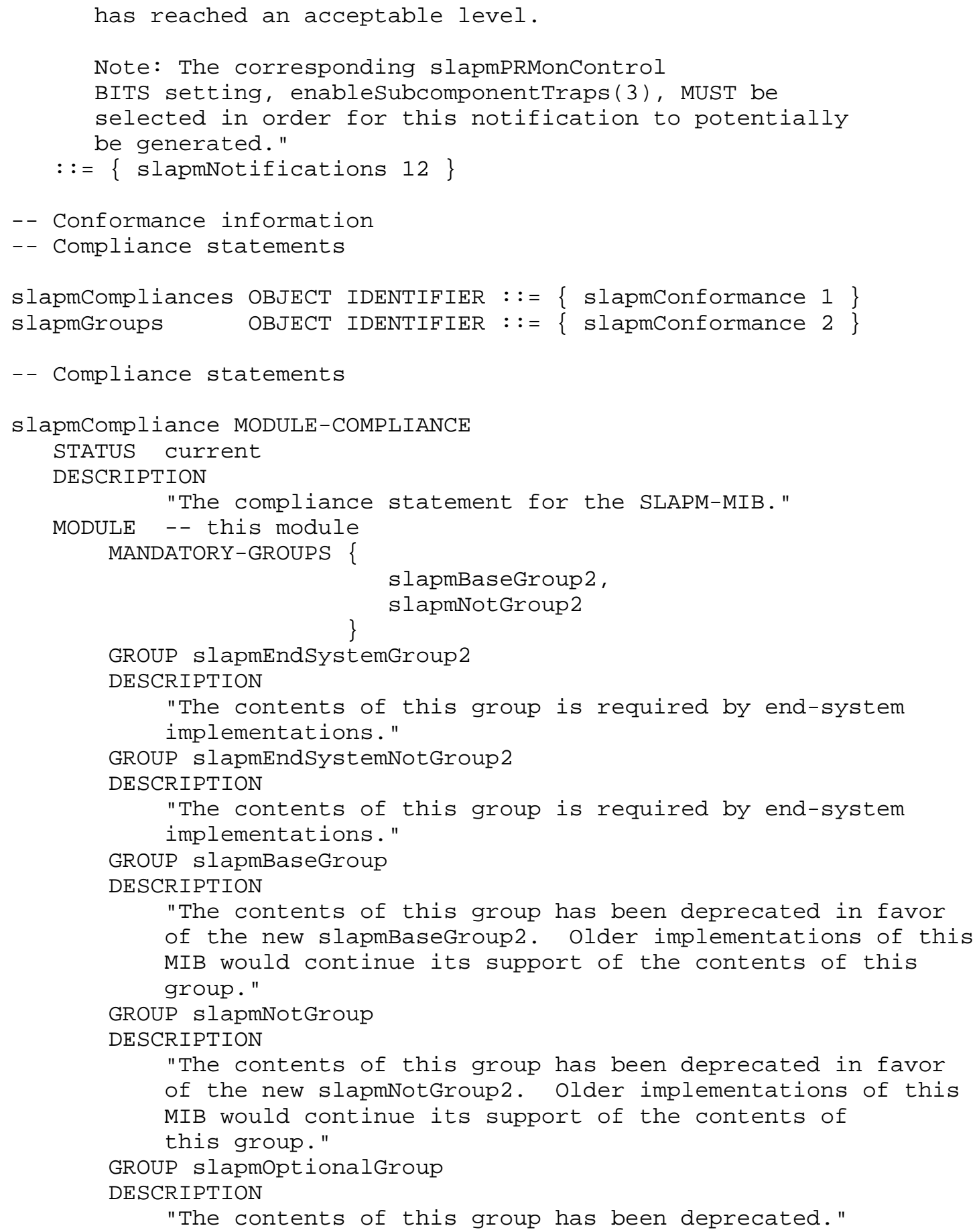




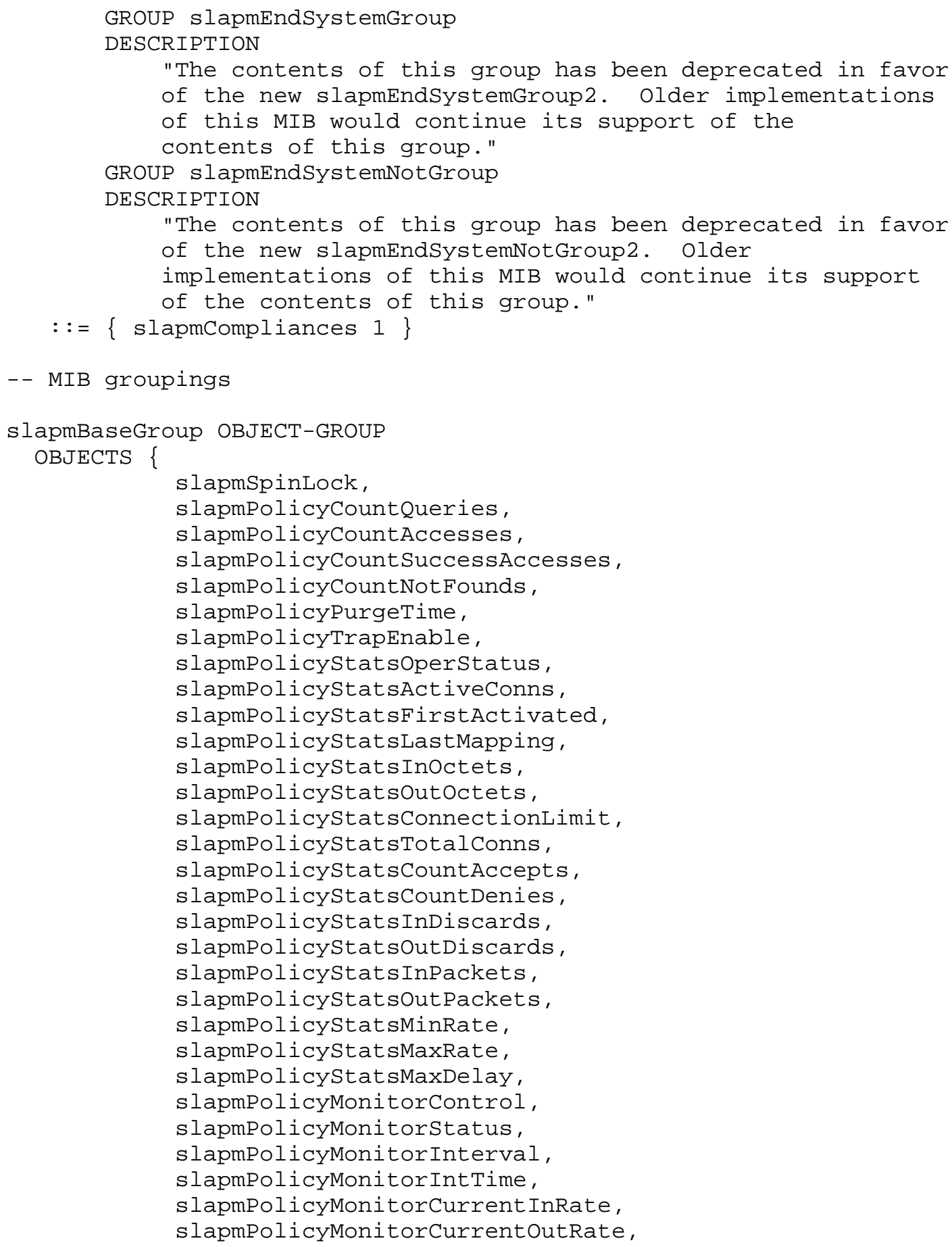




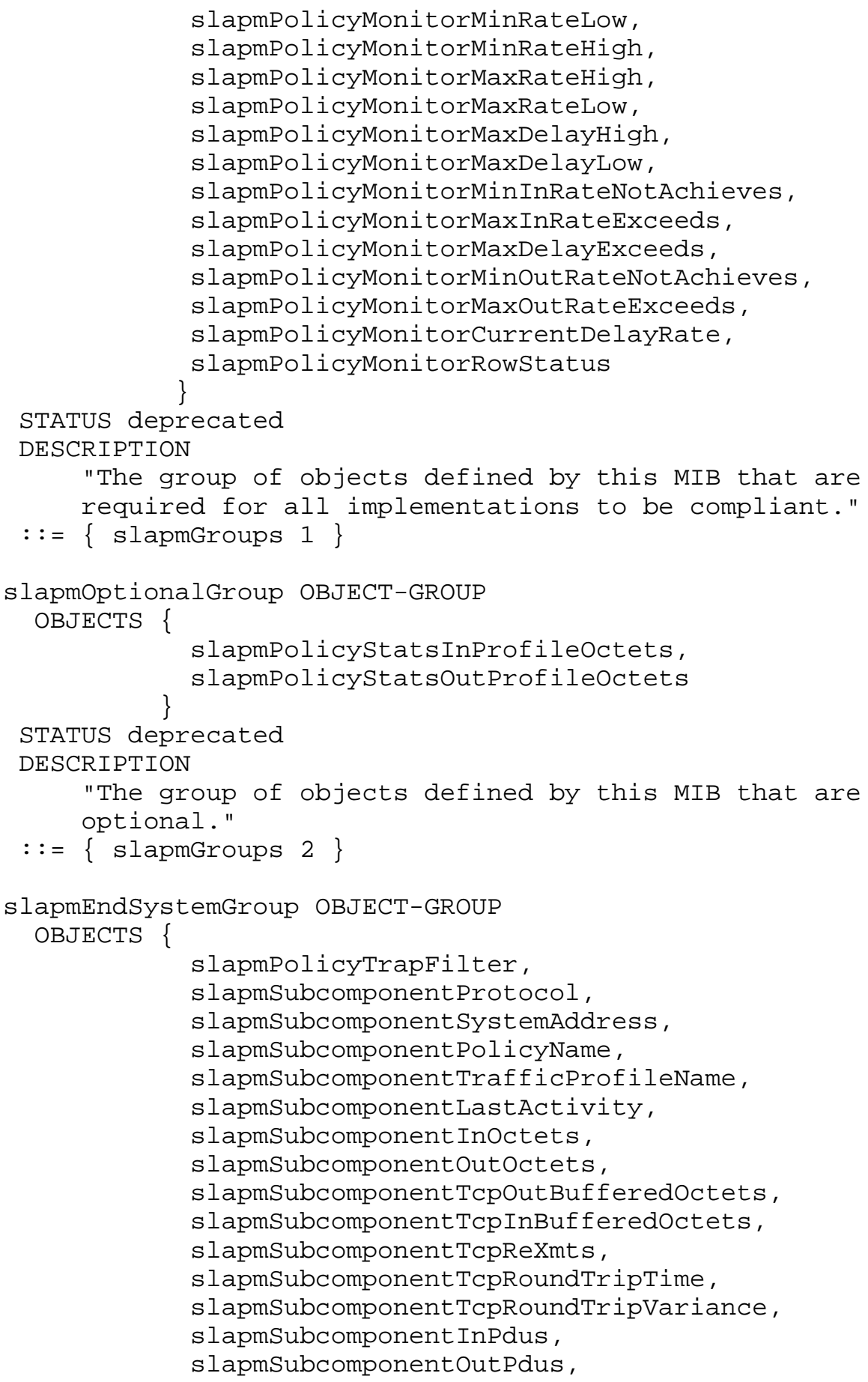




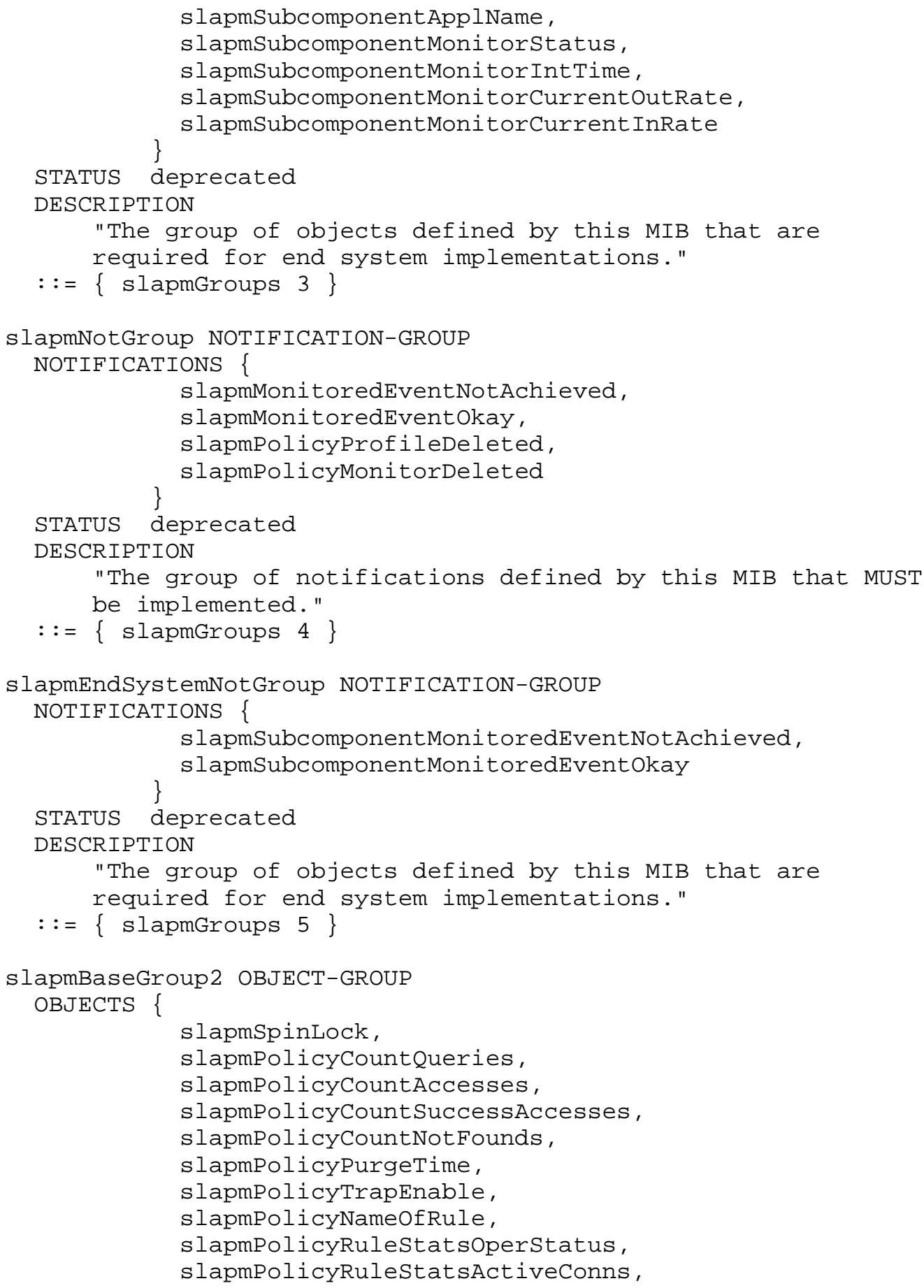




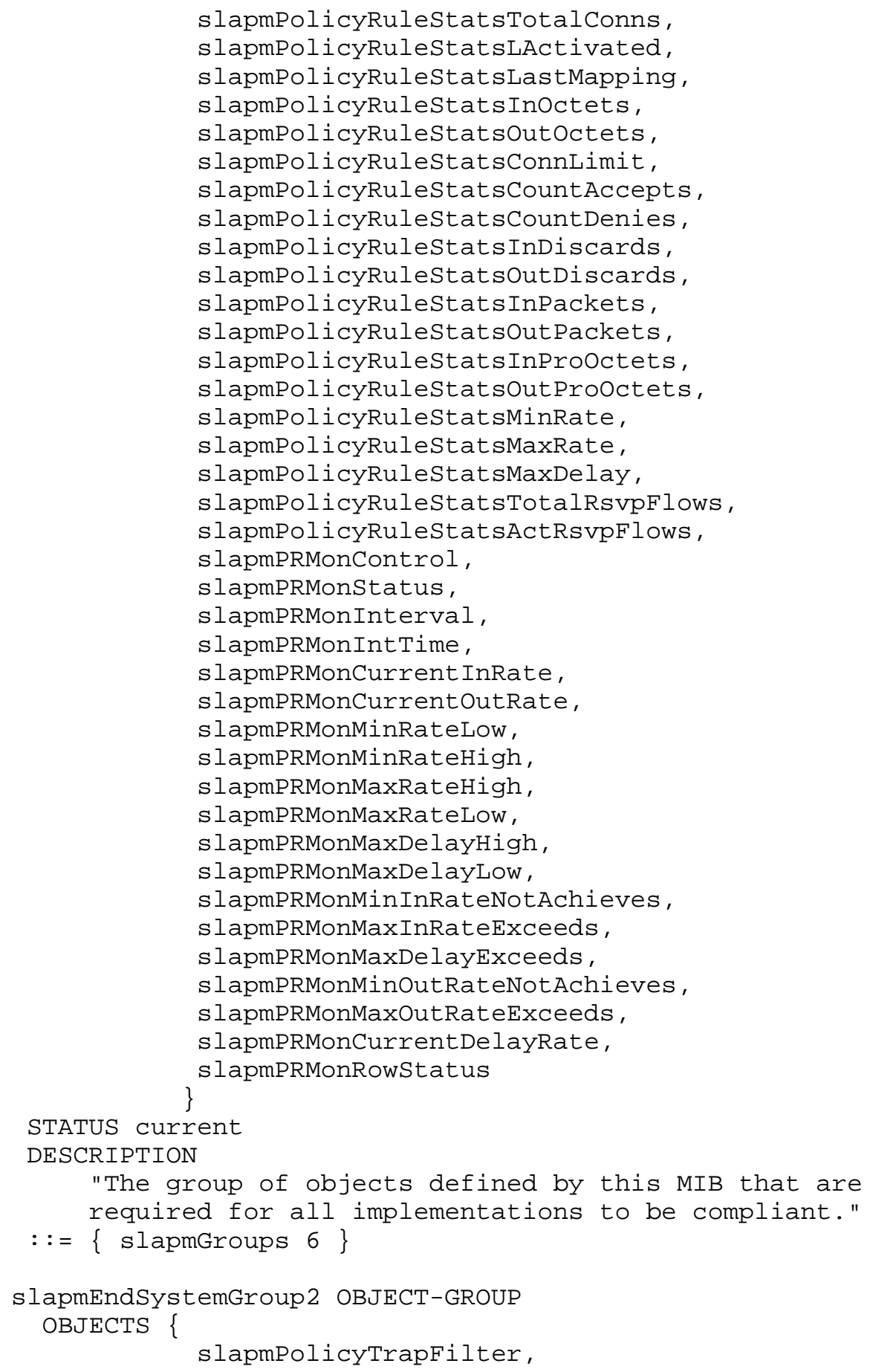




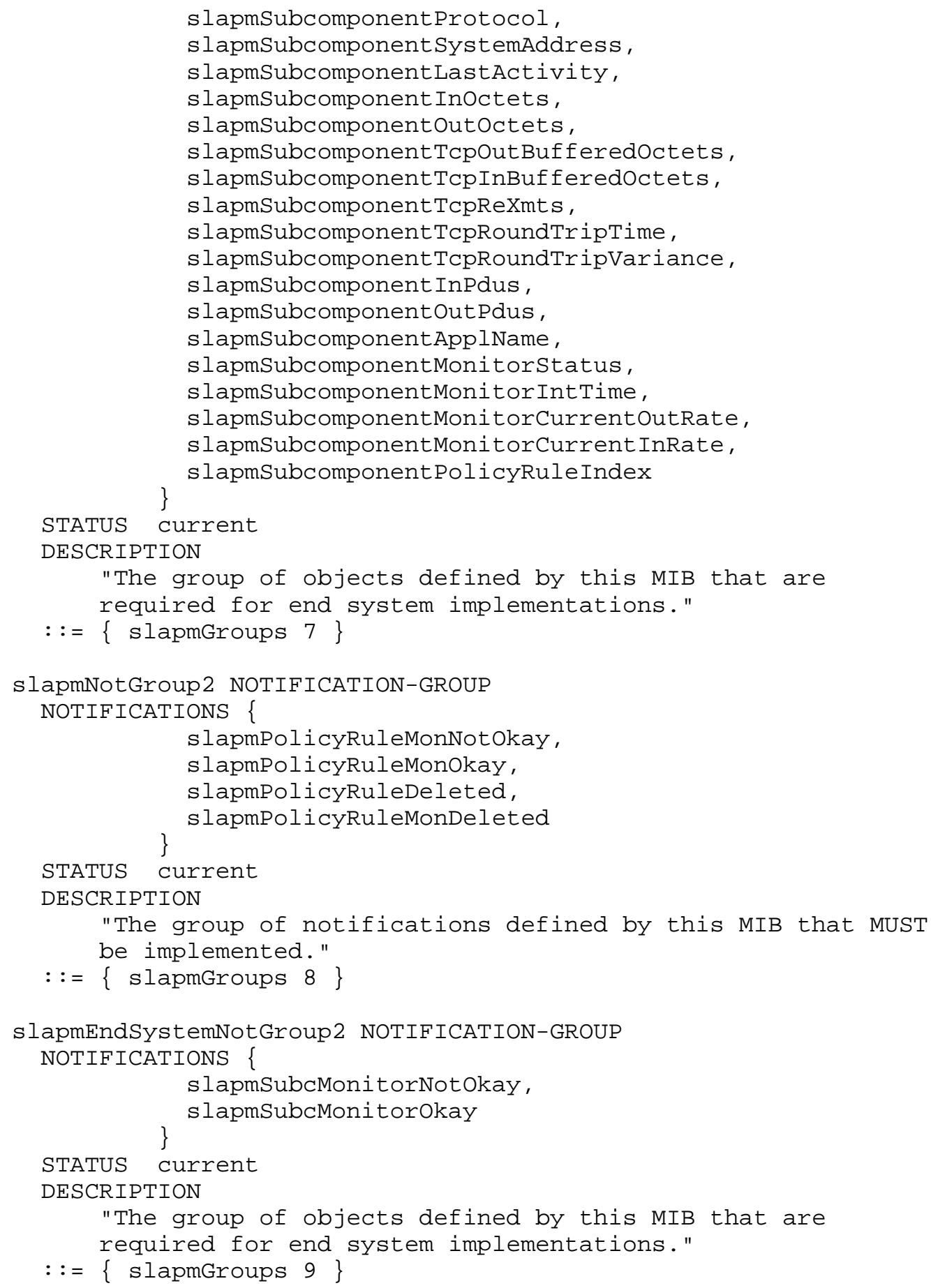


END

\subsection{Security Considerations}

Certain management information in the MIB defined by this document may be considered sensitive in some network environments. Therefore, authentication of received SNMP requests and controlled access to management information SHOULD be employed in such environments. The method for this authentication is a function of the SNMP Administrative Framework, and has not been expanded by this MIB.

To facilitate the provisioning of access control by a security administrator using the View-Based Access Control Model (VACM) defined in RFC 2575 [11] for tables in which multiple users may need to independently create or modify entries, the initial index is used as an "owner index" (refer to slapmPRMonOwnerIndex in an slapmPRMonEntry). Such an initial index has a syntax of SnmpAdminstring, and can thus be trivially mapped to a securityName or groupName as defined in VACM, in accordance with a security policy.

All entries in related tables belonging to a particular user will have the same value for this initial index. For a given user's entries in a particular table, the object identifiers for the information in these entries will have the same subidentifiers (except for the "column" subidentifier) up to the end of the encoded owner index. To configure VACM to permit access to this portion of the table, one would create vacmViewTreeFamilyTable entries with the value of vacmViewTreeFamilysubtree including the owner index portion, and vacmViewTreeFamilyMask "wildcarding" the column subidentifier. More elaborate configurations are possible. The VACM access control mechanism described above provides control

It is RECOMMENDED that the slapmPRMonTable (equivalent to the deprecated slapmPolicyMonitorTable) and the slapmSubcomponentTable not be supported in insecure environments.

\subsection{Intellectual Property}

The IETF takes no position regarding the validity or scope of any intellectual property or other rights that might be claimed to pertain to the implementation or use of the technology described in this document or the extent to which any license under such rights might or might not be available; neither does it represent that it has made any effort to identify any such rights. Information on the IETF's procedures with respect to rights in standards-track and standards-related documentation can be found in BCP-11. Copies of claims of rights made available for publication and any assurances of 
licenses to be made available, or the result of an attempt made to obtain a general license or permission for the use of such proprietary rights by implementers or users of this specification can be obtained from the IETF Secretariat.

The IETF invites any interested party to bring to its attention any copyrights, patents or patent applications, or other proprietary rights which may cover technology that may be required to practice this standard. Please address the information to the IETF Executive Director.

\subsection{Acknowledgments}

This document is an individual submission and not the product of any IETF working group. Special thanks should be given to Robert Moore of IBM for his numerous reviews.

\subsection{References}

[1] Case, J., Fedor, M., Schoffstall, M. and J. Davin, "Simple Network Management Protocol", STD 15, RFC 1157, May 1990.

[2] McCloghrie, K. and M. Rose, Editors, "Management Information Base for Network Management of TCP/IP-based internets: MIB-II", STD 17, RFC 1213, March 1991.

[3] McCloghrie, K., Perkins, D., Schoenwaelder, J., Case, J., Rose, M. and S. Waldbusser, "Structure of Management Information Version 2 (SMIV2)", STD 58, RFC 2578, April 1999.

[4] McCloghrie, K., Perkins, D., Schoenwaelder, J., Case, J., Rose, M. and S. Waldbusser, "Textual Conventions for SMIv2", STD 58, RFC 2579, April 1999.

[5] McCloghrie, K., Perkins, D., Schoenwaelder, J., Case, J., Rose, M. and S. Waldbusser, "Conformance Statements for SMIv2", STD 58, RFC 2580, April 1999.

[6] Case, J., McCloghrie, K., Rose, M. and Waldbusser, S., "Protocol Operations for Version 2 of the Simple Network Management Protocol (SNMPv2)", RFC 1905, January 1996.

[7] Harrington D., Presuhn, R. and B. Wijnen, "An Architecture for Describing SNMP Management Frameworks", RFC 2571, April 1999.

[8] Case, J., Harrington D., Presuhn, R. and B. Wijnen, "Message Processing and Dispatching for the Simple Network Management Protocol (SNMP)", RFC 2572, April 1999. 
[9] Levi D., Meyer P. and B. Stewart, "SNMPv3 Applications", RFC 2573, April 1999.

[10] Blumenthal, U. and B. Wijnen, "User-based Security Model (USM) for version 3 of the Simple Network Management Protocol (SNMPv3)", RFC 2574, April 1999.

[11] Wijnen, B., Presuhn, R. and K. McCloghrie, "View-based Access Control Model (VACM) for the Simple Network Management Protocol (SNMP) ", RFC 2575, April 1999.

[12] Hovey, R. and S. Bradner, "The Organizations Involved in the IETF Standards Process", BCP 11, RFC 2028, October 1996.

[13] Bradner, S., "Key words for use in RFCs to Indicate Requirement Levels", BCP 14, RFC 2119, March 1997.

[14] Rose, M. and K. McCloghrie, "Structure and Identification of Management Information for TCP/IP-based Internets", STD 16, RFC 1155, May 1990.

[15] Rose, M. and K. MCCloghrie, "Concise MIB Definitions", STD 16, RFC 1212, March 1991.

[16] Rose, M., "A Convention for Defining Traps for use with the SNMP", RFC 1215, March 1991.

[17] Case, J., McCloghrie, K., Rose, M. and S. Waldbusser, "Introduction to Community-based SNMPv2", RFC 1901, January 1996.

[18] Case, J., McCloghrie, K., Rose, M. and S. Waldbusser, "Transport Mappings for Version 2 of the Simple Network Management Protocol (SNMPv2) ", RFC 1906, January 1996.

[19] MCCloghrie, K. and A. Bierman, "Entity MIB using SMIv2", RFC 2037, October 1996.

[20] Bradner, S., "The Internet Standards Process -- Revision 3", BCP 9, RFC 2026, October 1996. 
9.0 Author's Address

Kenneth D. White

Dept. BRQA/Bldg. 501/G114

IBM Corporation

P.O.Box 12195

3039 Cornwallis

Research Triangle Park, NC 27709, USA

EMail: wkenneth@us.ibm.com 


\subsection{Full Copyright statement}

Copyright (C) The Internet Society (2000). All Rights Reserved.

This document and translations of it may be copied and furnished to others, and derivative works that comment on or otherwise explain it or assist in its implementation may be prepared, copied, published and distributed, in whole or in part, without restriction of any kind, provided that the above copyright notice and this paragraph are included on all such copies and derivative works. However, this document itself may not be modified in any way, such as by removing the copyright notice or references to the Internet society or other Internet organizations, except as needed for the purpose of developing Internet standards in which case the procedures for copyrights defined in the Internet Standards process must be followed, or as required to translate it into languages other than English.

The limited permissions granted above are perpetual and will not be revoked by the Internet society or its successors or assigns.

This document and the information contained herein is provided on an "AS IS" basis and THE INTERNET SOCIETY AND THE INTERNET ENGINEERING TASK FORCE DISCLAIMS ALL WARRANTIES, EXPRESS OR IMPLIED, INCLUDING BUT NOT LIMITED TO ANY WARRANTY THAT THE USE OF THE INFORMATION HEREIN WILL NOT INFRINGE ANY RIGHTS OR ANY IMPLIED WARRANTIES OF MERCHANTABILITY OR FITNESS FOR A PARTICULAR PURPOSE.

Acknowledgement

Funding for the RFC Editor function is currently provided by the Internet society. 